\title{
Selecting a Public Service Outsourcer Based on the Improved ELECTRE II Method with Unknown Weight Information under a Double Hierarchy Hesitant Linguistic Environment
}

\author{
Zhengmin Liu* ${ }^{\mathbb{D}}$, Xiaolan Zhao, Lin Li, Xinya Wang, Di Wang and Peide Liu \\ School of Management Science and Engineering, Shandong University of Finance and Economics, \\ Jinan 250014, China; 182106018@mail.sdufe.edu.cn (X.Z.); 182106017@mail.sdufe.edu.cn (L.L.); \\ Wangxinya@mail.sdufe.edu.cn (X.W.); Wangdi@mail.sdufe.edu.cn (D.W.); liupd@sdufe.edu.cn (P.L.) \\ * Correspondence: 1zm525@sdufe.edu.cn
}

Received: 10 February 2020; Accepted: 10 March 2020; Published: 16 March 2020

check for updates

\begin{abstract}
In present-day society, government public service outsourcing has become an irreversible trend due to the gradually increasing public pursuit of service quality and efficiency. To better meet the needs of the public and effectively improve the quality of service, it has been a crucial issue for government departments to choose the most desirable one from a series of public service outsourcers (PSOs) with distinct characteristics. In this paper, to deal with such decision problems, we propose the improved elimination and choice translating reality (ELECTRE) II method with unknown weight information under the double hierarchy hesitant fuzzy linguistic (DHHFL) environment to accurately and effectively select the best PSO. Firstly, aiming at the shortcomings of the original comparison method for double hierarchy hesitant fuzzy linguistic elements (DHHFLEs), we define the hesitant deviation degree (HDD) for DHHFLEs and, based on this, further propose a new comparison method for comparing DHHFLEs more reasonably. Secondly, inspired by the classical power average (PA) operator proposed by Yager, a new method is introduced to determine the weights of experts with respect to each attribute, based on the support degree between attributes. Afterwards, an improved ELECTRE II method is proposed to address the problem of PSO selection. A numerical case about e-government outsourcer selection is given to demonstrate the enforceability of the method. Finally, comparisons between previous methods and our method are carried out to illustrate the effectiveness and strengths of the proposed method.
\end{abstract}

Keywords: public service outsourcing; double hierarchy hesitant fuzzy linguistic term set; weight-determination method; ELECTRE II; multi-criteria decision-making

\section{Introduction}

Over the last two decades, to alleviate business pressure and focus on core competencies, firms are increasingly utilizing not only their own technologies, but also external resources and technologies [1-4], which is referred as open innovation (OI). Later, the OI phenomenon rapidly spread into many industries, and has received more and more attention [5-8]. As a form of OI, outsourcing has become a key component for business performance and has quickly expanded into the field of public services. Government public service outsourcing aims to enlist private organizations into the public service sector through a competitive market mechanism to compensate for government failures, reduce administrative costs, and improve service quality and efficiency. At the same time, considering that the contractors, who belong to the public sector and are the users of public services, can better understand the demands of the public, the government public service outsourcing can also build 
harmonious relationships among the government, the PSOs, and the public through the intermediary of the supplier. That is, by outsourcing public services to the service suppliers, the government can enhance both the economic effectiveness and the public satisfaction in line with the service target.

Driven by friendly policies, public service outsourcing has been rapidly promoted, and has gradually expanded from basic service areas, such as public infrastructure and solid waste management [9], to high-level public service areas, such as online IT service [10], energy supply [11], medical care [12], and social security [13]. As outsourced contractors, private organizations have the obligation to produce high-quality and efficient public services. The internal management level, scale, experience, and service capability of the contractor can directly determine the quality of public services, select the most efficient contractors, and standardize the contractors. When purchasing services and selecting the most appropriate service providers, the key to guaranteeing the quality of public services is to screen and inspect the service capabilities of contractors.

Regarding different service outsourcing providers as alternatives and their performances as criteria, respectively, the public service outsourcer (PSO) selection issue can then be identified as a typical multi-criteria decision-making (MCDM) problem [14]. The first and most basic step to solving the multi-attribute decision-making problem is to gather the assessment information given by the decision makers (DMs) about the alternatives with respect to the criteria. Due to the variability of environments and the complexity of people's cognition, uncertainty has become one of the characteristics of the decision-making field. In addition, as the selection of PSOs involves qualitative criteria, such as user experience and reliability, it is difficult for DMs to express evaluation information with accurate real numbers. Therefore, reasonably describing the expression of uncertain information has caused extensive concern from researchers. In this case, the fuzzy set [15] arose at the historic moment and has been continuously extended to different forms, such as type-2 fuzzy sets [16], intuitionistic fuzzy sets [17], and hesitant fuzzy sets [18]. Compared with precise values, quantitative linguistic information can more accurately depict the complicacy and fuzziness in the evaluation process. For instance, when assessing the user experience of a service provider, qualitative descriptions like "good" and "perfect" are commonly used rather than crisp numbers. From this perspective, various different linguistic representation models have been proposed to express the evaluation information of DMs, such as hesitant fuzzy linguistic term sets (HFLTSs) [19], the 2-tuple linguistic mode [20], and probability linguistic term sets [21].

Although various linguistic representation models have been applied to outsourcer selection, the abovementioned linguistic models cannot exactly depict the evaluation information given by experts, such as "between a little bad and very bad" and "between entirely moderate and a little good". Therefore, to better describe such linguistic evaluation information, based on the concept of HFLTSs, Gou et al. [22] further introduced the definition of double hierarchy hesitant linguistic term sets (DHHLTSs), which are made up of two hierarchy linguistic term sets (LTSs) in which the internal hierarchy LTS is used to make a further description or elaborate explanation for every LT contained in the external hierarchy LTS. DHHFLTSs are pretty useful tools in managing situations where DMs deliberate between several complex LTs when presenting their views on alternatives in the course of decision-making; this has become a project of widespread concern to researchers. Recently, Gou et al. [23] presented a number of distance and similarity measures of double hierarchy hesitant fuzzy linguistic (DHHFL) elements (DHHFLEs) and investigated a method for tackling the MCDM problems based on these measures. Taking the interactive features among criteria and the incomplete preference information into consideration, Liu et al. [24] developed a DHHFL mathematical programming means to handle the multi-attribute group decision-making problems. Gou et al. [25] proposed a consensus-reaching process for large-scale group decision making, in which the preference relations are described in the form of DHHFLEs. Krishankumar [26] constructed a novel decision framework in the context of DHHFLTS and proposed a new hybrid aggregation operator to aggregate the expert's preference under the DHHFL environment. 
The MCDM method plays an important role in MCDM and directly affects the accuracy and rationality of decision-making results. A great number of scholars have meticulously conducted in-depth research on outsourcer selection from this perspective; many classical decision-making methods and their extensions were applied to tackle the problem of outsourcer selection [27-30]. Among the methods applied to the problem of outsourcer selection, the elimination and choice translating reality (ELECTRE) method is a representative outranking method, originally proposed by Benayoun et al. [31], which has a distinct advantage in tackling the issue with non-compensatory criteria. After being meticulously probed and extended by a range of specialists, the method further evolved into the ELECTRE family, including ELECTRE I, ELECTRE II, ELECTRE III, and so on. Among them, the ELECTRE I method is suitable for constructing a partial prioritization and for choosing a set of promising alternatives [32], the ELECTRE III method aims to distribute each potential alternative into one of the categories in a previously defined family [33]. Compared with them, the ELECTRE II method [34] is a more pragmatic approach for handling the problem of ranking alternatives from the best to the worst $[35,36]$, which is more suitable for selecting the outsourcer in practical applications. In recent years, the ELECTRE II method has attracted wide attention from academia and has been extended into various forms under different fuzzy environments [37-40]. For example, Wan et al. [41] proposed the interval 2-tuple linguistic ELECTRE II to handle situations with non-compensatory criteria. Liao et al. [42] introduced two distinct ELECTRE II methods to deal with MCDM problems in line with the hesitant fuzzy linguistic terms set. Additionally, Lin et al. [43] investigated a new ELECTRE II method under the probabilistic linguistic environment. Nevertheless, the ELECTRE II method has not been extended to the DHHFL environment.

Based on the above literature review and analysis, we found the gaps in and insufficiency of the existing research. This paper is devoted to revising the comparison rule of DHHFLEs, extending the ELECTRE II method to the DHHFL environment, and further proposing a new ELECTRE II method combined with a novel weight-determination method. We implement our research through the following three points:

1. Considering the hesitation and complexity of experts' cognition in the problem of outsourcer selection, the DHHFLTS is introduced to express the evaluation information of experts for a given service provider. In addition, we found that the existing comparison method for DHHFLEs may provide erroneous results of comparisons in some cases. Hence, we proposed the concept of a hesitant deviation degree for DHHFLEs and developed a new comparison method for DHHFLEs based on this.

2. Inspired by the power average (PA) operator, we defined the support degree of the DHHFLEs and proposed a new weight determination method to determine the expert's weight for each criterion based on this, which can not only weaken the influence of extreme values on the results, but also reflects the expert's authority for each criterion.

3. The classical ELECTRE II method is extended to the DHHFL environment and then an improved ELECTRE II method is proposed, which has a distinct advantage in tackling issues with non-compensatory criteria, in order to select the most desirable PSOs. In this method, the proposed comparison method for DHHFLEs is used to identify the magnitude relationship between two alternatives under each criterion.

The rest of the article is organized as follows: Section 2 firstly reviews the corresponding concepts of the DHHFLTS and introduces the theoretical basis of the ELECTRE methods. Section 3 defines the concept of the hesitant deviation degree and further proposes a new comparison approach for DHHFLEs. Section 4 develops a new weight-determination method for experts with respect to each criterion and constructs a novel DHHFL-ELECTRE II method based on the new weight-determination method and the comparison method. Section 5 applies the novel ELECTRE II to pick out the e-government outsourcing provider. Section 6 carries out a comparative analysis to illustrate the effectiveness and 
strengths of our proposed method. Finally, Section 7 comes to a conclusion for this paper and indicates interesting directions for the following research work.

\section{Preliminaries}

To enhance the readability of this paper, the main symbols involved in the following formulas are summarized in Table 1.

Table 1. Related symbols involved in formulas and their descriptions.

\begin{tabular}{|c|c|c|c|}
\hline Symbols & Description & Symbols & Description \\
\hline$t$ & The subscript of the LT in outer LTS & $\kappa$ & The subscript of the LT in inner LTS \\
\hline$\varepsilon$ & The maximum subscript of the outer LTS & $w_{\xi}(k)$ & $\begin{array}{c}\text { The weight of the } k \text {-th expert under the } \\
\text { criterion } \mathrm{cr}_{\xi}\end{array}$ \\
\hline$\iota$ & The maximum subscript of the inner LTS & $\omega_{\xi}$ & The weight of criterion $c r_{\xi}$ \\
\hline$N *$ & The set of positive integers & $\omega_{c}$ & The attitude weight of concordance \\
\hline$\Delta$ & The number of elements in DHHFLE & $\omega=$ & The attitude weight of indifference \\
\hline$\Upsilon$ & The optimized parameter & $\omega_{d}$ & The attitude weight of discordance \\
\hline$\rho$ and $\rho^{-1}$ & $\begin{array}{c}\text { Transformation functions between the } \\
\text { real numbers and the elements in } \\
\text { DHHFLEs }\end{array}$ & $\xi$ & Lagrange multiplier \\
\hline
\end{tabular}

\subsection{DHHFLTS}

DHHFLTS, initially presented by Gou et al. [22], consists of two nested hierarchies of LTSs. The LTS of the outer hierarchy is given to convey the rough evaluation in the form of an adjective, like "moderate", "perfect", and "terrible". The inner-hierarchy LTS generally appeals in adverbs, such as "a little bit", "much", and so forth, which aim at describing the extent of the corresponding linguistic term (LT) in the outer hierarchy. In other words, the inner hierarchy can be regarded as a supplemental decoration to the outer. These two complementary hierarchies are expressed by two distinct LTSs with symmetric linguistic subscripts: $S^{f}=\left\{\zeta_{t} \mid t=-\varepsilon, \ldots,-1,0,1, \ldots, \varepsilon\right\}$ and $\phi^{\mathcal{S}}=\left\{\delta_{\kappa} \mid \kappa=-\iota, \ldots,-1,0,1, \ldots, \iota\right\}$, in which $\varepsilon$ and $\iota$ act as the scales of these two LTSs, respectively, and $\varepsilon, \iota \in N *$.

In view of the aforementioned theory, $S_{\phi}=\left\{\zeta_{t<\delta_{k}>} \mid t=-\varepsilon, \ldots,-1,0,1, \ldots, \varepsilon ; \kappa=-\iota, \ldots,-1,0,1, \ldots, l\right\}$ is defined as the double hierarchy linguistic term set (DHLTS), in which $\delta_{\mathcal{K}}$ stands for the homologous LT in the inner hierarchy, while the outer-hierarchy LT is denoted by $\zeta_{t}$. Accordingly, the intuitive mathematical expression of DHHFLTS can be given as:

$$
H_{S_{\phi}}=\left\{<\chi_{i}, h_{S_{\phi}}\left(\chi_{i}\right)>\mid \chi_{i} \in X\right\}
$$

where $h_{S_{\phi}}\left(\chi_{i}\right)$ is composed of several possible elements in $S_{\phi}$, which can be expressed as $h_{S_{\phi}}\left(\chi_{i}\right)=$ $\left\{\zeta_{t_{\delta}<\delta_{k_{\delta}}>} \mid \zeta_{t_{\delta}<\delta_{k_{\delta}}>} \in S_{\phi}, \delta=1,2, \ldots, \Delta h_{S_{\phi}}\right\}$ with $\Delta h_{S_{\phi}}$ being the amount of elements in $h_{S_{\phi}}\left(\chi_{i}\right)$. For the sake of convenience, $\zeta_{t<\delta_{k}}>$ is termed as the double hierarchy linguistic element (DHLE) and $h_{S_{\phi}}\left(\chi_{i}\right)$ is called the DHHFL element (DHHFLE). Considering that when the LT in the outer hierarchy is positive, the evaluation tends to be more positive as the degree of decorative adverbs deepens; when the LT in the outer hierarchy is negative, the evaluation tends to be more negative as the degree of decorative adverbs deepens. On the other hand, for LTs at both ends, only one-sided decorative adverbs are valid. Therefore, the inner-hierarchy LTS may be distinct under different LTs in the outer hierarchy: When $t \geq 0$, the degree of the elements contained in the outer-hierarchy LTS should be deepened with the increase of the subscript. On the contrary, when $t \leq 0$, as the subscript increases, the degree of the LTs contained in the outer-hierarchy LTS should be weakened. Hereinafter, suppose that both hierarchy LTSs are balanced, which means that semantic differences between any two adjacent LTs are equal. Considering the valence of linguistic terms, i.e., the emotional direction of linguistic terms, Figure 1 is given by Gou et al. [22] to elaborate the abovementioned four distinct shapes of the inner-hierarchy LTS in the case of $\iota=\varepsilon=3$. 


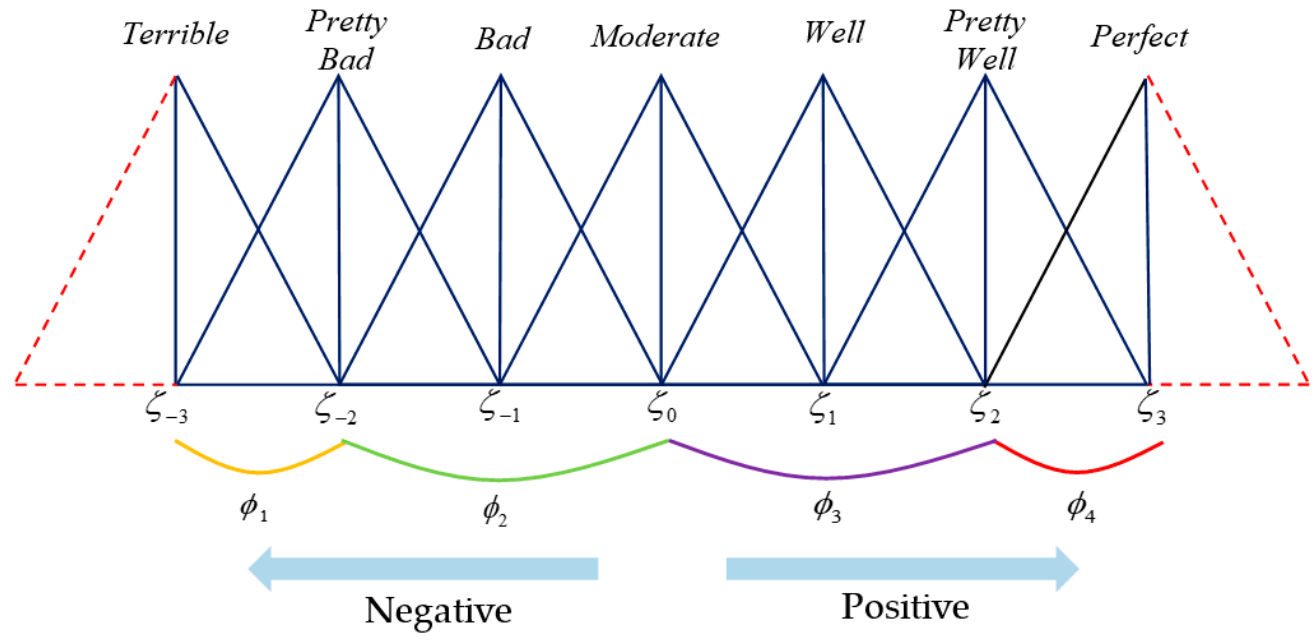

\footnotetext{
$\phi_{1}=\left\{\delta_{0}=\right.$ exactly right,$\delta_{1}=$ somewhat $\delta_{2}=$ a little bit,$\delta_{3}=$ far from $\}$

$\phi_{2}=\left\{\delta_{-3}=\right.$ entirely; $\delta_{-2}=$ very much; $\delta_{-1}=$ much $; \delta_{0}=$ exactly right $\delta_{1}=$ somewhat $\delta_{2}=$ a little bit,$\delta_{3}=$ far from $\}$

$\phi_{3}=\left\{\delta_{-3}=\right.$ far from; $\delta_{-2}=$ a little bit, $\delta_{-1}=$ somewhat $\delta_{0}=$ exactly right,$\delta_{1}=$ much; $\delta_{2}=$ very much; $\delta_{3}=$ entirely $\}$

$\phi_{4}=\left\{\delta_{-3}=\right.$ far from; $\delta_{-2}=$ a little bit $\delta_{-1}=$ somewhat,$\delta_{0}=$ exactly right $\}$
}

Figure 1. Four distinct shapes of the inner-hierarchy LTS.

To simplify the calculation, DHLEs and real numbers can be converted into each other through the following function introduced by Gou et al. [22].

$$
\begin{aligned}
& \rho:[-\varepsilon, \varepsilon] \times[-\iota, l] \rightarrow[0,1], \\
& \rho\left(\zeta_{t_{m}<\varphi_{k_{m}}>}\right)=\left\{\begin{array}{l}
\frac{1}{\varepsilon} \times \frac{\kappa_{m}+\iota}{2 l}+\frac{\varepsilon+t_{m}-1}{2 \varepsilon}=\frac{\kappa_{m}+\left(t_{m}+\varepsilon\right) \iota}{2 \varepsilon l}=\gamma_{m}, \text { if }-\varepsilon+1 \leq t_{m} \leq \varepsilon-1 . \\
\frac{1}{2 \varepsilon} \times \frac{\kappa_{m}+\iota}{2 \varepsilon}+\frac{\varepsilon+m_{m}-1}{2 \varepsilon}=\frac{\kappa_{m}+\left(t_{m}+\varepsilon\right) \iota}{2 \varepsilon l}=\gamma_{m}, \text { if } t_{m}=\varepsilon . \\
\frac{1}{2 \varepsilon} \times \frac{\kappa_{m}}{l}=\frac{\kappa_{m}}{2 \varepsilon l}=\gamma_{m}, \text { if } t_{m}=-\varepsilon .
\end{array}\right. \\
& \rho^{-1}:[0,1] \rightarrow[-\varepsilon, \varepsilon] \times[-\iota, \iota],
\end{aligned}
$$

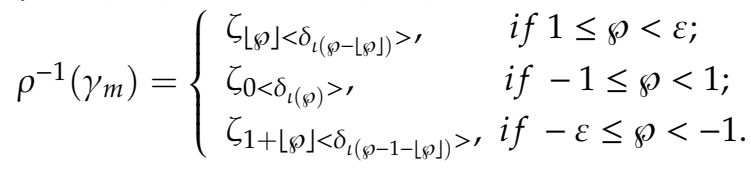

where $\left.\wp=2 \varepsilon \gamma_{m}-\varepsilon, L\right\rfloor$ is a round-down function that will returns the largest integer no more than the parameter. $\zeta_{t_{m}<\varphi_{k_{m}}>}$ and $\gamma_{m}$ represent the $m$-th elements in the DHHFLE and the hesitant fuzzy number, respectively. Obviously, since the last two formulas are special cases of the first one in Equation (2), it can be simplified to the following function:

$$
\begin{aligned}
& \rho:[-\varepsilon, \varepsilon] \times[-\iota, \iota] \rightarrow[0,1], \\
& \rho\left(\zeta_{t_{m}<\varphi_{k_{m}}>}\right)=\frac{1}{\varepsilon} \times \frac{\kappa_{m}+\iota}{2 \iota}+\frac{\varepsilon+t_{m}-1}{2 \varepsilon}=\frac{\kappa_{m}+\left(t_{m}+\varepsilon\right) \iota}{2 \varepsilon l}=\gamma_{m} .
\end{aligned}
$$

Gou et al. [22] extended the above conversion functions into a hesitant environment and presented the following conversion functions between the DHHFLEs and the hesitant fuzzy information, respectively.

$$
\begin{gathered}
I\left(h_{S_{\phi}}=\left\{\zeta_{t_{m}<\delta_{k_{m}}>} \mid \zeta_{t_{m}<\delta_{k_{m}}>} \in S_{\phi} ; t=[-\varepsilon, \varepsilon] ; \kappa=[-l, \iota] ; m=1,2, \ldots, \Delta h_{S_{\phi}}\right\}\right), \\
=\left\{\gamma_{m} \mid \gamma_{m}=\rho\left(t_{m}, \kappa_{m}\right)\right\}=h_{\gamma}
\end{gathered}
$$




$$
\begin{aligned}
I^{-1}\left(h_{\gamma}\right)=I^{-1}\left(\left\{\gamma_{m} \mid \gamma_{m} \in\right.\right. & {\left.\left.[0,1] ; m=1,2, \ldots, \Delta h_{\gamma}\right\}\right) } \\
& =\left\{\zeta_{t_{m}<\delta \kappa_{m}}>t_{m}<\delta_{\kappa_{m}}>=\rho^{-1}\left(\gamma_{m}\right)\right\}=h_{S_{\phi}} .
\end{aligned}
$$

Given any two DHHFLEs $h_{S_{\phi 1}}=\left\{\zeta^{1} t_{m}<\delta_{\kappa_{m}}>\mid \zeta_{t_{m}<\delta_{\kappa_{m}}>}^{1} \in S_{\phi} ; t=[-\varepsilon, \varepsilon] ; \kappa=[-\iota, l] ; m=1,2, \ldots, \Delta h_{S_{\phi 1}}\right\}$ and $h_{S_{\phi 2}}=\left\{\zeta_{t_{q}<\delta_{\kappa_{q}}>} \mid \zeta_{t_{q}<\delta_{\kappa_{q}}>} \in S_{\phi} ; t=[-\varepsilon, \varepsilon] ; \kappa=[-l, l] ; q=1,2, \ldots, \Delta h_{S_{\phi 2}}\right\}$, the essential operations are given as:

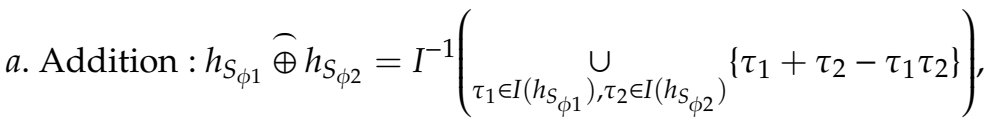

$$
\begin{aligned}
& \text { b. Mutiplication : } h_{S_{\phi 1}} \widehat{\otimes} h_{S_{\phi 2}}=I^{-1}\left(\tau_{\tau_{1} \in I\left(h_{S_{\phi 1}}\right), \tau_{2} \in I\left(h_{S_{\phi 2}}\right)}\left\{\tau_{1} \tau_{2}\right\}\right) \text {, } \\
& \text { c. } N \text {-mutiplication : } \psi \cdot h_{S_{\phi 1}}=I^{-1}\left(\bigcup_{\tau \in I\left(h_{S_{\phi 1}}\right)}\left\{1-(1-\tau)^{\psi}\right\}\right) \text {, } \\
& \text { d. Power : } h_{S_{\phi 1}} \psi=I^{-1}\left(\bigcup_{\tau \in I\left(h_{S_{\phi 1}}\right)}\left\{\tau^{\psi}\right\}\right) \text {, } \\
& \text { e. Inverse : }\left(h_{S_{\phi 1}}\right)^{c}=I^{-1}\left(\bigcup_{\tau \in I\left(h_{S_{\phi 1}}\right)}\{1-\tau\}\right) \text {. }
\end{aligned}
$$

The distance measure involved in fuzzy evaluation information is a crucial concept in decision theory, and it can be exploited to reflect the inconsistency degree between two distinct information numbers. Given two DHHFLEs $h_{S_{\phi 1}}$ and $h_{S_{\phi 2}}$, the Hamming distance between them, defined by Gou et al. [23], is found as below:

$$
\begin{aligned}
& h d\left(h_{S_{\phi 1}}, h_{S_{\phi 2}}\right)=\frac{1}{\Delta h} \quad \sum_{m=1}^{\Delta h} \quad\left|u_{m}-v_{m}\right|, \\
& u_{m}=\rho\left(\zeta_{t_{m}<\delta_{\kappa_{m}}>}\right) \\
& v_{m}=\rho\left(\zeta_{t_{m}<\delta_{\kappa_{m}}>}^{2}\right)
\end{aligned}
$$

where $\Delta h$ is the number of elements in $h_{S_{\phi 1}}$ and $h_{S_{\phi 2}}$ (here suppose $\Delta h_{S_{\phi 1}}=\Delta h_{S_{\phi 2}}=\Delta h$ ), $\zeta^{1} t_{m}<\delta_{\kappa_{m}}>$ and $\zeta_{t_{m}<\delta_{\kappa_{m}}}^{2}$ indicate the $m$-th optimal values in $h_{S_{\phi 1}}$ and $h_{S_{\phi 2}}$.

Remark 1. Considering that the number of DHLEs is not always the same, Gou et al. [23] proposed a method to expand the shorter DHHFLE by adding a double hierarchy LTS to it until $\Delta h_{S_{\phi 1}}=\Delta h_{S_{\phi 2}}$ : Given a DHHFLE $h_{S_{\phi}}=\left\{\zeta_{t_{m}<\delta_{\kappa_{m}}>}>\zeta_{t_{m}<\delta_{\kappa_{m}}>} \in S_{\phi} ; t=[-\varepsilon, \varepsilon] ; \kappa=[-l, l] ; m=1,2, \ldots, \Delta h_{S_{\phi}}\right\}$, with $\zeta_{t_{1}<\delta_{\kappa_{1}}>}$ and $\zeta_{t_{\Delta h_{S_{\phi}}}}<\delta_{\kappa_{\Delta h_{S_{\phi}}}}>$ being the minimum and maximum DHLE in it, respectively, then, we can expand $h_{S_{\phi}}$ with the following DHLE:

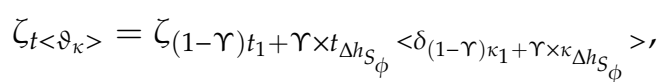

where $\checkmark$ is an optimized parameter satisfying $0 \leq \checkmark \leq 1$, which is used to reflect the risk preference of the DM. The larger the $\checkmark$ is, the higher the risk preference of the DM enjoys. In this paper, we assume that the DM is risk neutral, i.e., $\checkmark=1 / 2$; then, the DHLE that we can add to the DHHFLE is $\zeta_{t<\vartheta_{\kappa}>}=\frac{\zeta_{t_{1}+t_{\Delta h_{\phi}}}}{2} \frac{\vartheta_{\kappa_{1}+\kappa_{\Delta h} h_{\phi}}}{2}$.

\subsection{Main Concepts in ELECTRE Methods}

This subsection is devoted to showing two main theoretical supports of the ELECTRE method: The binary outranking relations and the definitions of concordance and discordance. 
Definition 1. [44]. The expression of preferences in the ELECTRE methods depends on a basic binary priority relation, $S$, which means "at least as good as". Based on this fundamental relation, four mutually exclusive situations might arise when comparing two alternatives e and $f$ as follows:

1. eSf and not $f S e$, which means "e is strictly preferred to $f$ ", i.e., ePf;

2. fSe and not eSf, which means " $f$ is strictly preferred to $e$ ", i.e., $f P e$;

3. eSf and fSe, which means "e is indifferent to $f$ ", i.e., elf;

4. Neither eSf nor fSe, which means "e and f are not comparable", i.e., eRf.

Definition 2. [44]. All of the outranking methods stand by the ideas of concordance and discordance, which indicate, respectively, the augments of supporting and opposing an outranking relation:

1. Concordance: For the given binary outranking relation eSf, concordance refers to the combined power of the criteria in favor of this outranking. To implement eSf, there must be as much of a majority as possible of criteria supporting this inference.

2. Discordance: Discordance reflects the combined power of the criteria against the outranking eSf. Generally, a convictive outranking should be opposed by as few criteria as possible.

\section{The New Comparison Method for DHHFLEs}

\subsection{Existing Comparison Method}

To measure the performance of DHHFLEs, Gou et al. [22] provided the definition of the expected value and variance for DHHFLEs as follows:

Given a DHHFLE $h_{S_{\phi}}=\left\{\zeta_{t_{m}<\delta_{\kappa_{m}}>} \mid \zeta_{t_{m}<\delta_{\kappa_{m}}>} \in S_{\phi} ; t=[-\varepsilon, \varepsilon] ; \kappa=[-\iota, \iota] ; m=1,2, \ldots, \Delta h_{S_{\phi}}\right\}$ with $\Delta h_{S_{\phi}}$ being the number of elements in $h_{S_{\phi}}$, then the mathematical form of its expected value $\stackrel{\leftrightarrow}{E} v\left(h_{S_{\phi}}\right)$ and variance $\stackrel{\leftrightarrow}{V} a\left(h_{S_{\phi}}\right)$ can be given as:

$$
\begin{gathered}
\stackrel{\leftrightarrow}{E} v\left(h_{S_{\phi}}\right)=\frac{1}{\Delta h_{S_{\phi}}} \sum_{r=1}^{\Delta h_{S_{\phi}}} \rho\left(\zeta_{t_{r}<\delta_{\kappa_{r}}}\right), \\
\stackrel{\leftrightarrow}{V} a\left(h_{S_{\phi}}\right)=\sqrt{\frac{1}{\Delta h_{S_{\phi}}} \sum_{r=1}^{\Delta h_{S_{\phi}}}\left(\rho\left(\zeta_{t_{r}<\delta_{\kappa_{r}}}\right)-\stackrel{\leftrightarrow}{E} v\left(h_{S_{\phi}}\right)\right)^{2}},
\end{gathered}
$$

where $\rho$ is the conversion function between the real number and the DHLE.

Based on the functions of the expected value and the variance, Gou et al. [22] proposed a comparison method for any two DHHFLEs, which pointed out that the DHHFLE with lower variance is superior to that with high variance when their expected values are equal. However, this is not always the truth. In the following, an example is given to explain the irrationality of this method.

Example 1. Given two DHHFLES $h_{S_{\phi 1}}=\left\{\zeta_{-1<\delta_{-2}>}, \zeta_{0<\delta_{-1}>}, \zeta_{0}, \zeta_{0<\delta_{1}>}, \zeta_{1<\delta_{2}>}\right\}$ and $h_{S_{\phi 2}}=\left\{\zeta_{-1<\delta_{-2}>}, \zeta_{0}, \zeta_{1<\delta_{2}>}\right\}$, based on Equations (14) and (15), we can obtain $\stackrel{\leftrightarrow}{E} v\left(h_{S_{\phi 1}}\right)=0.5, \stackrel{\leftrightarrow}{E} v\left(h_{S_{\phi 2}}\right)=0.5$, $\stackrel{\leftrightarrow}{V} a\left(h_{S_{\phi 1}}\right)=0.179$ and $\stackrel{\leftrightarrow}{V} a\left(h_{S_{\phi 2}}\right)=0.226$, respectively. According to the comparison method proposed by Gou et al., $h_{S_{\phi 1}}$ is superior to $h_{S_{\phi 2}}$ on account of $\stackrel{\leftrightarrow}{E} v\left(h_{S_{\phi 1}}\right)=\stackrel{\leftrightarrow}{E} v\left(h_{S_{\phi 2}}\right)$ and $\stackrel{\leftrightarrow}{V} a\left(h_{S_{\phi 1}}\right)<\stackrel{\leftrightarrow}{V} a\left(h_{S_{\phi 2}}\right)$. Nonetheless, from a cognitive point of view, under the condition that the expected values of the two DHHFLEs are equal and that the maximum and minimum values of the elements in them are likewise equal, the more elements there are in the DHHFLE, the more hesitant the DM is, and the poorer the performance of the DHHFLE will be. Therefore, on this occasion, the conclusion obtained by Gou et al.'s comparison method is unreasonable. 
Obviously, Gou et al. only took expectation and variance into consideration, but ignored the impact of the number of elements in DHHFLE. Therefore, based on the variance, we take the hesitant degree into consideration and put forward the definition of the hesitant-deviation degree (HDD) of a DHHFLE.

\subsection{The Improved Comparison Method}

Definition 3. Let $h_{S_{\phi}}=\left\{\zeta_{t_{m}<\delta_{\kappa m}}>\zeta_{t_{m}<\delta_{\kappa_{m}}>} \in S_{\phi} ; t=[-\varepsilon, \varepsilon] ; \kappa=[-l, l] ; m=1,2, \ldots, \Delta h_{S_{\phi}}\right\}$ be a DHHFLE, then the HDD of $h_{S_{\phi}}$ can be expressed by

$$
h \sigma\left(h_{S_{\phi}}\right)=\frac{\Delta h_{S_{\phi}}-1}{2 \varepsilon \tau} \sqrt{\frac{1}{\Delta h_{S_{\phi}}} \sum_{r=1}^{\Delta h_{S_{\phi}}}\left(\rho\left(\zeta_{t_{r}<\delta_{\kappa_{r}}}\right)-\stackrel{\leftrightarrow}{E} v\left(h_{S_{\phi}}\right)\right)^{2}}
$$

where $\Delta h_{S_{\phi}}$ is the number of elements in $h_{S_{\phi}}$, and $\left(\Delta h_{S_{\phi}}-1\right) / 2 \varepsilon \tau$ is used to reflect the hesitant degree of a DHHFLE. Obviously, the greater the number of elements in $h_{S_{\phi}}$, the bigger the hesitant degree is, i.e., the greater the uncertainty is. $\sqrt{\frac{1}{\Delta h_{S_{\phi}}} \sum_{r=1}^{\Delta h_{S_{\phi}}}\left(\rho\left(\zeta_{t_{r}<\delta_{\kappa_{r}}}\right)-\stackrel{\leftrightarrow}{E} v\left(h_{S_{\phi}}\right)\right)^{2}}$ denotes the variance function shown in Equation (15), which is used to reflect the deviation degree of a DHHFLE. Obviously, in Equation (16), the hesitant degree and deviation degree of the DHHFLE are simultaneously taken into account, which comprehensively reflects the uncertainty and discreteness of the elements in the DHHFLE. For a DHHFLE, a greater HDD indicates a weaker consistency and instability.

Next, a new comparison method for DHHFLEs is put forward based on Equations (14) and (16).

Definition 4. Given any two DHHFLEs $h_{S_{\phi 1}}$ and $h_{S_{\phi 2}}$, then:

(1) Suppose $\stackrel{\leftrightarrow}{E} v\left(h_{S_{\phi 1}}\right)>\stackrel{\leftrightarrow}{E} v\left(h_{S_{\phi 2}}\right)$, then $h_{S_{\phi 1}}$ is superior to $h_{S_{\phi 2}}$.

(2) Suppose $\stackrel{\leftrightarrow}{E} v\left(h_{S_{\phi 1}}\right)=\stackrel{\leftrightarrow}{E} v\left(h_{S_{\phi 2}}\right)$, then

Suppose $h \sigma\left(h_{S_{\phi 1}}\right)<h \sigma\left(h_{S_{\phi 2}}\right)$, then $h_{S_{\phi 1}}$ is superior to $h_{S_{\phi 2}}$;

Suppose $h \sigma\left(h_{S_{\phi 1}}\right)=h \sigma\left(h_{S_{\phi 2}}\right)$, then $h_{S_{\phi 1}}$ is indifferent to $h_{S_{\phi 2}}$.

Example 2. For the two DHHFLEs $h_{S_{\phi 1}}=\left\{\zeta_{-1<\delta_{-2}>}, \zeta_{0<\delta_{-1}>}, \zeta_{0}, \zeta_{0<\delta_{1}>}, \zeta_{1<\delta_{2}>}\right\}$ and $h_{S_{\phi 2}}=\left\{\zeta_{-1<\delta_{-2}>}, \zeta_{0}\right.$, $\left.\zeta_{1<\delta_{2}>}\right\}$ mentioned in Example 1, we can obtain that $h \sigma\left(h_{S_{\phi 1}}\right)=0.039, h \sigma\left(h_{S_{\phi 2}}\right)=0.019$ by using Equation (16). For $\stackrel{\leftrightarrow}{E} v\left(h_{S_{\phi 1}}\right)=\stackrel{\leftrightarrow}{E} v\left(h_{S_{\phi 2}}\right)$ and $h \sigma\left(h_{S_{\phi 1}}\right)>h \sigma\left(h_{S_{\phi 2}}\right)$, we can draw the accurate conclusion that $h_{S_{\phi 2}}$ is superior to $h_{S_{\phi 1}}$ on the basis of our proposed comparison method. Apparently, with the hesitant degree taken into consideration, the new comparison is more reasonable and effective than that introduced by Gou et al.

\section{The Improved Expected-HDD-ELECTRE II Method}

\subsection{Problem Characterization}

Consider an MCDM that involves $m$ alternatives expressed by $Z=\left\{o p_{1}, o p_{2}, \ldots, o p_{m}\right\}$, a group of experts denoted by $E=\left\{e x_{1}, e x_{2}, \ldots, e x_{g}\right\}$, and $n$ attributes expressed as $C=\left\{c r_{1}, c r_{2}, \ldots, c r_{n}\right\}$. The weight vectors of experts and criteria are completely unknown, owing to the information asymmetry of the real problem. To accurately describe their opinions, the experts take the form of DHHFLE $h_{S_{\phi i j}}$ 
to express their evaluations of the $\mathrm{i}$-th option with respect to the criterion $\mathrm{j}$. Then, the decision matrix of the expert $e x_{\vartheta}$ is given as follows:

$$
\mathbf{R}^{(\vartheta)}=\left[h_{S_{\phi i j}}(\vartheta)\right]_{m \times n}=\left[\begin{array}{llll}
h_{S_{\phi 11}}(\vartheta) & h_{S_{\phi 12}}(\vartheta) & \cdots & h_{S_{\phi 1 n}}(\vartheta) \\
h_{S_{\phi 21}}(\vartheta) & h_{S_{\phi 22}}(\vartheta) & \cdots & h_{S_{\phi 2 n}}(\vartheta) \\
\vdots & \vdots & \ddots & \vdots \\
h_{S_{\phi m 1}(\vartheta)} & h_{S_{\phi m 2}}(\vartheta) & \cdots & h_{S_{\phi m n}}(\vartheta)
\end{array}\right], \vartheta=1,2, \ldots, g .
$$

\subsection{Determining the Expert's Weight with Respect to Each Attribute}

Among the existing weight determination methods, one of the familiar reference values that they rely on is the distance or similarity between assessments and the mean values, which are vulnerable to extreme values and may lead to inaccurate results. In addition, the research fields of experts might be so various that there are some differences in the knowledge, skills, and experiences of each expert. An expert may be an expert in certain attributes, but not in other attributes. Considering these two points, we present a novel approach to determine the expert's weight regarding each attribute based on the degrees of support to each other. Then the steps are as follows.

Step 1: To facilitate subsequent calculations, we combine all of the decision matrices $R^{(\vartheta)}(\vartheta=$ $1,2, \ldots, g)$ provided by each expert and convert them into other matrices regarding each attribute, which can be denoted as shown below.

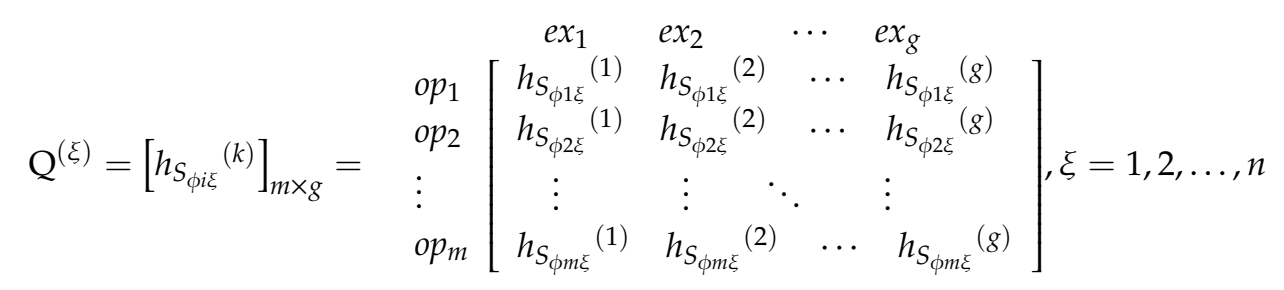

in which the $\vartheta$-column vector in $\mathrm{Q}^{(\xi)}$ is exactly the duplicate of the $\xi$-column vector in $\mathrm{R}^{(\vartheta)}$.

Step 2: Obtain the support degrees of $h_{S_{\phi i \xi}}{ }^{(p)}(p=1,2, \ldots, g)$ from $h_{S_{\phi i \xi}}{ }^{(q)}(q=1,2, \ldots, g ; q \neq p)$.

Inspired by the PA operator, the support degrees from $h_{S_{\phi i \xi}}(q)$ to $h_{S_{\phi i \xi}}(\xi)$ can be defined as follows:

$$
\sup \left(h_{S_{\phi i \xi}}(p), h_{S_{\phi i \xi}}(q)\right)=1-d\left(h_{S_{\phi i \xi}}(p), h_{S_{\phi i \xi}}(q)\right) .
$$

Obviously, the smaller the distance between $h_{S_{\phi i \xi}}(q)$ and $h_{S_{\phi i \xi}}(p)$, the greater the support degree from $h_{S_{\phi i \xi}}(q)$ to $h_{S_{\phi i \xi}}(p)$.

Step 3: Calculate the total support degree $T\left(h_{S_{\phi i \xi}}(p)\right)$ of $h_{S_{\phi i \xi}}(p)(p=1,2, \ldots, g)$ as

$$
T\left(h_{S_{\phi i \xi}}(p)\right)=\sum_{\substack{q=1 \\ q \neq p}}^{g} \sup \left(h_{S_{\phi i \xi}}(p), h_{S_{\phi i \xi}}(q)\right), i=1,2, \ldots, m ; \xi=1,2, \ldots, n .
$$

Apparently, a greater $T\left(h_{S_{\phi i \xi}}{ }^{(p)}\right)$ indicates a better similarity to others. From the compromise point of view, the DM whose evaluation value is close to the evaluation values of other experts, i.e., who enjoys a great total support degree, would have a big weight. 
Then, the total support degree matrix can be given as below:

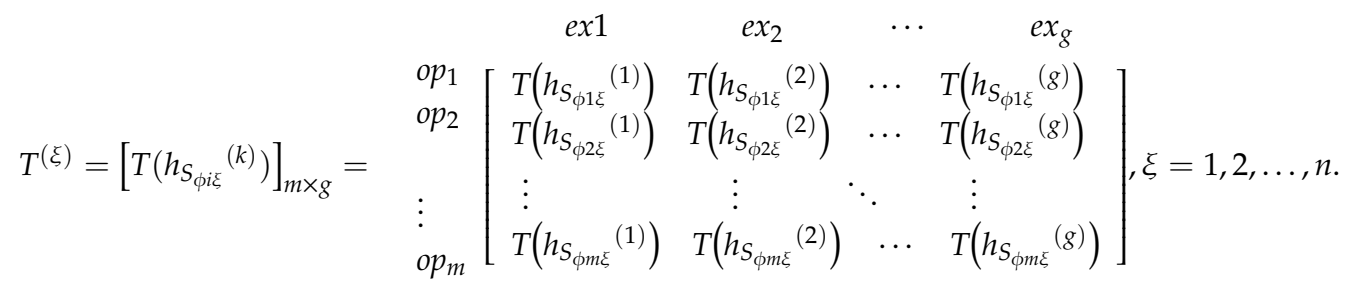

Step 4: Taking the sum of elements in each column of the matrix $T^{(\xi)}$, we can obtain the overall support degree of each expert regarding the criterion $\mathrm{Cr}_{\xi}$ by the following equation.

$$
l_{\xi}{ }^{(k)}=\sum_{i=1}^{m} T\left(h_{S_{\phi i \xi}}{ }^{(k)}\right), k=1,2, \ldots, g ; \xi=1,2, \ldots, n .
$$

Step 5: Obtain each expert's weight regarding the criterion $\mathrm{cr}_{\xi}$ by the following equation.

$$
w_{\xi}(k)=\frac{l_{\xi}(k)}{\sum_{t=1}^{g} l_{\xi}(t)}, k=1,2, \ldots, g .
$$

Apparently, $0 \leq w_{\xi}^{(k)} \leq 1$ and $\sum_{k=1}^{g} w_{\xi}^{(k)}=1$.

In conclusion, the novel weight-determination method for experts has two features or advantages: On one hand, this method, combined with the PA operator, leads to the definition of the total support degree to eliminate the influence of extreme values, which is more rational than other methods based on the average values or ideal solutions. On the other hand, the method aims to build a convergent position: For each expert, the weight may vary with different attributes, and the higher the total support degree from other experts, the greater the weight of this expert under this attribute. In this way, the unreasonable deviation caused by the expert's knowledge, skills, experience, and other factors can be avoided, and the decision process would be more precise and reasonable.

\subsection{Obtaining the Criterion's Weight Based on the Optimization Model}

After getting each DM's weight with respect to each attribute, an overall matrix $\mathbb{R}$ can be given as follows by aggregating all of the decision matrices $\mathrm{R}^{(\vartheta)}(\vartheta=1,2, \ldots, g)$.

$$
\mathrm{R}=\left[h_{S_{\phi i j}}\right]_{m \times n}=\left[\begin{array}{llll}
h_{S_{\phi 11}} & h_{S_{\phi 12}} & \cdots & h_{S_{\phi 1 n}} \\
h_{S_{\phi 21}} & h_{S_{\phi 22}} & \cdots & h_{S_{\phi 2 n}} \\
\vdots & \vdots & \ddots & \vdots \\
h_{S_{\phi m 1}} & h_{S_{\phi m 2}} & \cdots & h_{S_{\phi m n}}
\end{array}\right]
$$

In the present world, considering that decision making is always accompanied by uncertain settings and inconsistent opinions, most decisions are made from the perspective of compromise. Based on this point, Ju [45] presented a weight-determination method for criteria whose weights are completely unknown based on the optimization model and ideal solutions. Then, we apply it to the DHHFL environment; the steps are given as follows:

Step 1: Obtain the DHHFL's positive ideal solution (DHHFL-PIS) and the DHHFL's negative ideal solution (DHHFL-NIS) by Equations (24) and (25), respectively.

$$
h_{S_{\phi}}{ }^{+}=\left(h_{S_{\phi 1}}{ }^{+}, h_{S_{\phi 2}}{ }^{+}, \ldots, h_{S_{\phi n}}{ }^{+}\right),
$$




$$
h_{S_{\phi}}{ }^{-}=\left(h_{S_{\phi 1}}{ }^{-}, h_{S_{\phi 2}}{ }^{-}, \ldots, h_{S_{\phi n}}{ }^{-}\right) \text {, }
$$

where $h_{S_{\phi j}}{ }^{+}=\max _{i=1}^{m}\left\{h_{S_{\phi i j}}{ }^{+}\right\}, h_{S_{\phi j}}{ }^{-}=\min _{i=1}^{m}\left\{h_{S_{\phi i j}}{ }^{-}\right\}, j=1,2, \ldots, n$.

Step 2: Calculate the Hamming distance $h d\left(h_{S_{\phi i j}} h_{S_{\phi j}}{ }^{+}\right)(i=1,2, \ldots, m ; j=1,2, \ldots, n)$

Step 3: Calculate the Hamming distance $h d\left(h_{S_{\phi i j}} h_{S_{\phi j}}{ }^{-}\right)(i=1,2, \ldots, m ; j=1,2, \ldots, n)$.

Step 4: Establish a multiple objective optimization model and solve it to obtain the weight vector of the criteria.

$$
\begin{aligned}
h_{i}{ }^{+} & =\sum_{j=1}^{n} h d\left(h_{S_{\phi i j}} h_{S_{\phi j}}{ }^{+}\right) \times \omega_{j}, i=1,2, \ldots, m, \\
h_{i}{ }^{-}= & \sum_{j=1}^{n} h d\left(h_{S_{\phi i j}} h_{S_{\phi j}}{ }^{-}\right) \times \omega_{j}, i=1,2, \ldots, m .
\end{aligned}
$$

From the perspective of compromise, the greater the values of $h_{i}{ }^{+}$and $h_{i}{ }^{-}$are, the higher priority the alternate option $o p_{i}$ will be. In other words, an appropriate weight vector $\dddot{W}=\left(\omega_{1}, \omega_{2}, \ldots, \omega_{n}\right)$ should make the value of $h_{i}{ }^{+}$and $h_{i}{ }^{-}$as large as possible. Hence, a multiple objective optimization model can be established as below:

$$
(M 1)\left\{\begin{array}{l}
\max H=\sum_{i=1}^{m} \sum_{j=1}^{n}\left(\left(h d\left(h_{S_{\phi i j}} h_{S_{\phi j}}{ }^{+}\right)+h d\left(h_{S_{\phi i j}}, h_{S_{\phi j}}{ }^{-}\right)\right) \times \Theta_{j}\right) \\
\text { s.t. } \sum_{j=1}^{n} \omega_{j}^{2}=1, \omega_{j} \in N, j=1,2, \ldots, n
\end{array} .\right.
$$

To solve the optimization model and obtain the most desirable weight vector, a Lagrange function with a Lagrange multiplier $\xi$ can be given as:

$$
L(\dddot{W}, \xi)=\sum_{i=1}^{m} \sum_{j=1}^{n}\left(\left(h d\left(h_{S_{\phi i j}} h_{S_{\phi j}}{ }^{+}\right)+h d\left(h_{S_{\phi i j^{\prime}}} h_{S_{\phi j}}{ }^{-}\right)\right) \times \omega_{j}\right)+\frac{1}{2} \xi\left(\sum_{j=1}^{n} \omega_{j}{ }^{2}-1\right) .
$$

Taking the partial derivative of $L(\dddot{W}, \xi)$ with respect to $\omega_{j}$ and $\xi$ and setting them equal to zero, respectively, we can obtain the following equation:

$$
\left\{\begin{array}{l}
\frac{\partial L(\dddot{\mathcal{W}}, \zeta)}{\partial \omega_{j}}=\sum_{i=1}^{m}\left(h d\left(h_{S_{\phi i j}}, h_{S_{\phi j}}{ }^{+}\right)+h d\left(h_{S_{\phi i j}}, h_{S_{\phi j}}{ }^{-}\right)\right)+\zeta \cdot \omega_{j}=0, j=1,2, \ldots, n ; \\
\frac{\partial L(\dddot{W}, \zeta)}{\partial \zeta}=\frac{1}{2}\left(\sum_{j=1}^{n}{\omega_{j}}^{2}-1\right)=0 .
\end{array}\right.
$$

Settling the simultaneous equations above, we can obtain the weight of each criterion as follows:

$$
\omega_{j}^{\prime}=\frac{\sum_{i=1}^{m}\left(h d\left(h_{S_{\phi i j}}, h_{S_{\phi j}}{ }^{+}\right)+h d\left(h_{S_{\phi i j}}, h_{S_{\phi j}}{ }^{-}\right)\right)}{\sqrt{\sum_{j=1}^{n}\left(\sum_{i=1}^{m}\left(h d\left(h_{S_{\phi i j}} h_{S_{\phi j}}{ }^{+}\right)+h d\left(h_{S_{\phi i j}} h_{S_{\phi j}}\right)\right)\right)^{2}}}, j=1,2, \ldots, n .
$$

In real practical terms, the convention assumes that the criteria weights add up to 1 . Therefore, we make a further normalization for the criteria weight obtained by Equation (33) as follows:

$$
\omega_{j}=\frac{\omega_{j}^{\prime}}{\sum_{j=1}^{n} \omega_{j}^{\prime}}=\frac{\sum_{i=1}^{m}\left(h d\left(h_{S_{\phi i j}} h_{S_{\phi j}}{ }^{+}\right)+h d\left(h_{S_{\phi i j}} h_{S_{\phi j}}{ }^{-}\right)\right)}{\sum_{j=1}^{n} \sum_{i=1}^{m}\left(h d\left(\dot{h}_{S_{\phi i j}}, h_{S_{\phi j}}{ }^{+}\right)+h d\left(h_{S_{\phi i j}}, h_{S_{\phi j}}{ }^{-}\right)\right)}, j=1,2, \ldots, n .
$$


By using the calculated criterion's weight to weight the matrix $\mathrm{R}$, we can get the following final decision matrix:

$$
\overline{\mathrm{R}}=\left[\bar{h}_{S_{\phi i j}}\right]_{m \times n}=\left[\begin{array}{llll}
\bar{h}_{S_{\phi 11}} & \bar{h}_{S_{\phi 12}} & \ldots & \bar{h}_{S_{\phi 1 n}} \\
\bar{h}_{S_{\phi 21}} & \bar{h}_{S_{\phi 22}} & \ldots & \bar{h}_{S_{\phi 2 n}} \\
\vdots & \vdots & \ddots & \vdots \\
\bar{h}_{S_{\phi m 1}} & \bar{h}_{S_{\phi m 2}} & \ldots & \bar{h}_{S_{m n}}
\end{array}\right],
$$

where $\bar{h}_{S_{\phi i j}}=\omega_{j} \times h_{S_{\phi i j}}$.

\subsection{The Expected-HDD-Based ELECTRE II Method}

On the basis of the new comparison method for DHHFLEs and the weight-determination methods for experts and criteria, an improved ELECTRE II method is proposed to rank the given alternatives in the decision making with DHHFL information. In our method, on one hand, the improved comparison method for DHHFLEs is used to identify the magnitude relationship between two alternatives under each criterion, which can make the division of concordant, indifferent, and discordant sets more accurate. On the other hand, the novel weight-determination method for experts can eliminate the effects of both extremes and the experts' experience, which can provide more accurate information for the following steps of the method and makes the decision result more convincing.

\subsubsection{Concordant, Indifferent, and Discordant Sets}

In the traditional ELECTRE II method, by means of the comparisons of each pair of options on the attributes, the attributes of distinct alternate options can be grouped into three mutually exclusive collections: The concordant set $J^{+}=\left\{j \mid c r_{j}\left(o p_{s}\right)>c r_{j}\left(o p_{t}\right)\right\}$ indicating those attributes under which $o p_{s}$ is superior to $o p_{t}$, the indifference set $J^{=}=\left\{j \mid c r_{j}\left(o p_{s}\right)=c r_{j}\left(o p_{t}\right)\right\}$ indicating those attributes under which $o p_{s}$ is indiscriminate with $o p_{t}$ and the discordance set $J^{-}=\left\{j \mid c r_{j}\left(o p_{s}\right)<c r_{j}\left(o p_{t}\right)\right\}$ denoting those attributes under which $o p_{s}$ is inferior to $o p_{t}$, in which $c r_{j}\left(o p_{s}\right)$ represents the performance of the option $o p_{s}$ as regard to the criterion $c r_{j}$.

In the following, based the proposed comparison method for DHHFLEs, the expected value and HDD of the DHHFLEs are denoted together to distinguish the different alternatives under a certain criterion, which makes the division of attributes more sophisticated and reasonable. In view of the notions of the expected value and the HDD function for DHHFLEs, the DHHFL concordant set (DHHFLCS) and DHHFL discordant set (DHHFLDS) can be subdivided into the DHHFL strong, moderate, and weak concordant (discordant) sets, respectively.

For any pair of options $o p_{s}$ and $o p_{t}(s, t=1,2, \ldots, m ; s \neq t)$, the DHHFLCS of them is a collection of criteria under which $o p_{s}$ is superior to $o p_{t}$. It can be subdivided into three types, as shown below:

(1) The strong DHHFLCS $J c_{s t}{ }^{s}$ :

$$
J c_{s t}{ }^{s}=\left\{j \mid \stackrel{\leftrightarrow}{E} v\left(h_{S_{\phi s j}}\right)>\stackrel{\leftrightarrow}{E} v\left(h_{S_{\phi t j}}\right) \& h \sigma\left(h_{S_{\phi s j}}\right)<h \sigma\left(h_{S_{\phi t j}}\right)\right\}
$$

(2) The moderate DHHFLCS $J c_{s t}{ }^{m}$ :

$$
J c_{s t}{ }^{m}=\left\{j \mid \stackrel{\leftrightarrow}{E} v\left(h_{S_{\phi s j}}\right)>\stackrel{\leftrightarrow}{E} v\left(h_{S_{\phi t j}}\right) \& h \sigma\left(h_{S_{\phi s j}}\right) \geq h \sigma\left(h_{S_{\phi t j}}\right)\right\}
$$

(3) The weak DHHFLCS $J c_{s t}{ }^{w}$ :

$$
J c_{s t}{ }^{w}=\left\{j \mid \stackrel{\leftrightarrow}{E} v\left(h_{S_{\phi s j}}\right)=\stackrel{\leftrightarrow}{E} v\left(h_{S_{\phi t j}}\right) \& h \sigma\left(h_{S_{\phi s j}}\right)<h \sigma\left(h_{S_{\phi t j}}\right)\right\}
$$


These three DHHFLCSs indicate three distinct and decreasing extents of the concordance for the assertion " $o p_{s}$ is at least as good as $o p_{t}$ ". Obviously, the distinction between $J c_{s t}{ }^{s}$ and $J c_{s t}{ }^{m}$ is caused by the HDD. A lower HDD denotes a high stability degree of the DHHFLE; therefore, compared with $J c_{s t}{ }^{m}$, $J c_{s t}{ }^{s}$ represents a higher degree of concordance. According to Definition 4, a conclusion can easily be drawn that $h_{S_{\phi s j}}$ is superior to $h_{S_{\phi s j}}$ as long as $\stackrel{\leftrightarrow}{E} v\left(h_{S_{\phi s j}}\right)>\stackrel{\leftrightarrow}{E} v\left(h_{S_{\phi t j}}\right)$. In other words, the expected value plays a more important role than the HDD when comparing two DHHFLEs. Hence, $J c_{s t}{ }^{m}$ indicates a higher degree of concordance than $J c_{s t}{ }^{w}$.

In the same manner, the DHHFLDS indicates a collection of criteria under which $o p_{s}$ is inferior to $o p_{t}$. It can be subdivided into three types, as shown below:

(1) The strong DHHFLDS $J d_{s t}{ }^{s}$ :

$$
J d_{s t}{ }^{s}=\left\{j \mid \stackrel{\leftrightarrow}{E} v\left(h_{S_{\phi s j}}\right)<\stackrel{\leftrightarrow}{E} v\left(h_{S_{\phi t j}}\right) \& h \sigma\left(h_{S_{\phi s j}}\right)>h \sigma\left(h_{S_{\phi t j}}\right)\right\}
$$

(2) The moderate DHHFLDS $J d_{s t}{ }^{m}$ :

$$
J d_{s t}{ }^{m}=\left\{j \mid \stackrel{\leftrightarrow}{E} v\left(h_{S_{\phi s j}}\right)<\stackrel{\leftrightarrow}{E} v\left(h_{S_{\phi t j}}\right) \& h \sigma\left(h_{S_{\phi s j}}\right) \leq h \sigma\left(h_{S_{\phi t j}}\right)\right\}
$$

(3) The weak DHHFLDS $J d_{s t}{ }^{w}$ :

$$
J d_{s t}^{w}=\left\{j \mid \stackrel{\leftrightarrow}{E} v\left(h_{S_{\phi s j}}\right)=\stackrel{\leftrightarrow}{E} v\left(h_{S_{\phi t j}}\right) \& h \sigma\left(h_{S_{\phi s j}}\right)>h \sigma\left(h_{S_{\phi t j}}\right)\right\}
$$

In addition to the abovementioned conditions, there still stands a case where $\stackrel{\leftrightarrow}{E} v\left(h_{S_{\phi s j}}\right)=\stackrel{\leftrightarrow}{E} v\left(h_{S_{\phi t j}}\right)$ and $h \sigma\left(h_{S_{\phi s j}}\right)=h \sigma\left(h_{S_{\phi t j}}\right)$; the set of corresponding subscripts of these criteria is called the indifferent set, and can be defined as follows:

$$
J I_{s t}=\left\{j \mid \stackrel{\leftrightarrow}{E} v\left(h_{S_{\phi s j}}\right)=\stackrel{\leftrightarrow}{E} v\left(h_{S_{\phi t j}}\right) \& h \sigma\left(h_{S_{\phi s j}}\right)=h \sigma\left(h_{S_{\phi t j}}\right)\right\} .
$$

\subsubsection{The DHHFL Concordance and Discordance Index}

In line with the abovementioned DHHFLCS and DHHFLDS, for the assertion "the $o p_{s}$ is at least as good as the $o p_{t}{ }^{\prime \prime}$, the DHHFL concordance index (DHHFLCI) can be obtained by

$$
\begin{aligned}
& C I_{s t}=\frac{\omega_{c}^{s} \times \sum_{\sigma \in J_{s t} s^{s}} \omega_{\sigma}+\omega_{c}^{m} \times \sum_{\sigma \in J_{s t} t^{m}} \omega_{\sigma}+\omega_{c}{ }^{w} \times \sum_{\sigma \in J c_{s t} t^{w}} \omega_{\sigma}+\omega^{=} \times \sum_{\sigma \in I_{s t}} \omega_{\sigma}}{\sum_{\sigma=1}^{n} \omega_{\sigma}} \\
& =\omega_{c}{ }^{s} \times \sum_{\sigma \in J \mathcal{C}_{s t} t^{s}} \omega_{\sigma}+\omega_{c}{ }^{m} \times \sum_{\sigma \in J \mathcal{C}_{s t}{ }^{m}} \omega_{\sigma}+\omega_{c}{ }^{w} \times \sum_{\sigma \in J \mathcal{C}_{s t}{ }^{w}} \omega_{\sigma}+\omega^{=} \times \sum_{\sigma \in J I_{s t}} \omega_{\sigma}
\end{aligned}
$$

where $\omega_{\sigma}$ is the weight of the criterion $c r_{\sigma} . \omega_{c}{ }^{s}, \omega_{c}{ }^{m}, \omega_{c}{ }^{w}$ and $\omega^{=}$are the given position weights for the strong DHHFLCS, moderate DHHFLCS, weak DHHFLCS, and DHHFL indifferent set, respectively. The DHHFLCI $C I_{s t}$ indicates the weighted superior degree of the alternative $o p_{s}$ over the alternative $o p_{t}$, and $C I_{s t} \in[0,1]$. Then, we can construct the DHHFL concordant matrix as follows:

$$
C=\left[C I_{s t}\right]_{m \times m}=\left[\begin{array}{llll}
- & C I_{12} & \cdots & C I_{1 m} \\
C I_{21} & - & \cdots & C I_{2 m} \\
\vdots & \vdots & \ddots & \vdots \\
C I_{m 1} & C I_{m 2} & \cdots & -
\end{array}\right] .
$$


Similarly, the DHHFL discordance index (DHHFLDI) of DHHFLDS can be given as

$$
D I_{S t}=\frac{\max \left\{\omega_{d}^{s} \times \max _{\sigma \in J d_{s t} s}\left\{\omega_{\sigma} \cdot h d\left(h_{S_{\phi s \sigma}}, h_{S_{\phi t \sigma}}\right)\right\}, \omega_{d}^{m} \times \max _{\sigma \in J d_{s t} m}\left\{\omega_{\sigma} \cdot h d\left(h_{S_{\phi s \sigma}}, h_{S_{\phi t \sigma}}\right)\right\}, \omega_{d}^{w} \times_{\sigma \in J d_{s t} w^{w}}\left\{\omega_{\sigma} \cdot h d\left(h_{S_{\phi s \sigma}}, h_{S_{\phi t \sigma}}\right)\right\}\right\}}{\max _{j} h d\left(h_{S_{\phi s j}}, h_{S_{\phi t j}}\right)}
$$

where $\omega_{\sigma}$ is the weight of the criterion $c r_{\sigma} . \omega_{d}{ }^{s}, \omega_{d}{ }^{m}$ and $\omega_{d}{ }^{w}$ are the given attitude weights for the strong DHHFLDS, moderate DHHFLDS, and weak DHHFLDS, respectively. $h d\left(h_{S_{\phi s \sigma}}, h_{S_{\phi t \sigma}}\right)$ denotes the Hamming distance between $h_{S_{\phi s \sigma}}$ and $h_{S_{\phi t \sigma}}$, which can be obtained by Equation (6). The DHHFLDI DI $I_{s t}$ represents the weighted inferior degree of the alternative $o p_{s}$ over the alternative $o p_{t}$, and $D I_{s t} \in[0,1]$.

Likewise, the DHHFL discordant matrix can be established as:

$$
D=\left[D I_{s t}\right]_{m \times m}=\left[\begin{array}{lrrrr}
- & D I_{12} & \cdots & D I_{1 m} \\
D I_{21} & - & \cdots & D I_{2 m} \\
\vdots & \vdots & \ddots & \vdots & \\
D I_{m 1} & D I_{m 2} & \cdots & -
\end{array}\right]
$$

\subsubsection{Determine the Outranking Relations}

When the DHHFL concordant matrix and the DHHFL discordant matrix are determined, we can define the critical value $\bar{C}$ by taking the mean value of all the elements in the DHHFL concordant matrix, which can be expressed by $\bar{C}=\sum_{s=1}^{m} \sum_{t=1}^{m} C I_{s t} / m(m-1)$. Then, making the comparisons between the elements in the DHHFL concordant set and the critical value $\bar{C}$, respectively, the DHHFL concordant Boolean matrix can be obtained as follows:

$$
B_{C}=\left[b c_{s t}\right]_{m \times m}=\left[\begin{array}{cccc}
- & b c_{12} & \cdots & b c_{1 m} \\
b c_{21} & - & \cdots & b c_{2 m} \\
\vdots & \vdots & \ddots & \vdots \\
b c_{m 1} & b c_{m 2} & \cdots & -
\end{array}\right],
$$

where the element $b c_{s t}$ can only be 0 or 1 , and when $C I_{s t} \geq \bar{C}, b c_{s t}=1$; otherwise, $b c_{s t}=0 . b c_{s t}=1$ means that the alternative $o p_{s}$ outranks the alternative $o p_{t}$ from the perspective of concordance.

Similarly, a marginal value can be given by the average of all of the elements in the DHHFL discordant matrix, presented by $\bar{D}=\sum_{s=1}^{m} \sum_{t=1}^{m} D I_{s t} / m(m-1)$. Then, the discordant Boolean matrix is defined as below:

$$
B_{D}=\left[b d_{s t}\right]_{m \times m}=\left[\begin{array}{crcc}
- & b d_{12} & \cdots & b d_{1 m} \\
b d_{21} & - & \cdots & b d_{2 m} \\
\vdots & \vdots & \ddots & \vdots \\
b d_{m 1} & b d_{m 2} & \cdots & -
\end{array}\right] \text {, }
$$

where the element $b d_{s t}$ can only be 0 or 1 , and when $D I_{s t}<\bar{D}, b d_{s t}=1$; otherwise, $b d_{s t}=0 . b d_{s t}=1$ indicates that the alternative $o p_{s}$ is not inferior to the alternative $o p_{t}$ from the perspective of discordance.

Then, to show the overall outranking relations between the alternatives, a comprehensive Boolean matrix $B$ can be defined through making a multiplication of the Boolean matrices $B_{C}$ and $B_{D}$, which can be expressed as $B=\left[b c_{s t} \times b d_{s t}\right]_{m \times m}=\left[b_{s t}\right]_{m \times m}$. Obviously, only when $b c_{s t}$ and $b d_{s t}$ are both assigned of a value $1, b_{s t}=1$, which means that the option $o p_{s}$ is strictly outranking to the option $o p_{t}$.

\subsubsection{Draw the Outranking Graph}

According to the comprehensive Boolean matrix $B$ obtained in the last section, an outranking graph $G=(V, L)$ can be depicted to concisely show the outranking relations between alternatives and to help the DM choose the most desirable alternative. In the graph, $V$ is a set of vertices and each vertex 
represents an alternative, $L$ indicates the set of directed edges connecting the vertices, and each arc shows the outranking relation between the two alternatives at either end of it. There are two situations of the directed arcs between two alternatives $o p_{s}$ and $o p_{t}$ : (1) The directed edge is from $o p_{s}$ to $o p_{t}$, which indicates that $o p_{s}$ outperforms $o p_{t} ;(2)$ when the two alternatives $o p_{s}$ and $o p_{t}$ are incomparable, there is no arc between the two corresponding vertices.

In general, the symmetric positions in the matrix $B$ should be complementary, as the relation of strictly outranking cannot be mutual. In other words, there should be and merely is one arc between any two vertices in the outranking graph. However, owing to the subjectivity of the threshold selection, the symmetric positions in the comprehensive Boolean matrix $B$ are not always complementary, which makes it impossible to compare the two schemes. To handle this issue, Lin et al. [36] proposed the following method to revise the Boolean matrices.

Let $B$ be a comprehensive matrix obtained by the multiplication of the Boolean matrices $B_{C}$ and $B_{D}$ in component form. When the elements $B_{s t}=B_{t s}=0$ or $B_{s t}=B_{t s}=1$, which implies that the elements in the corresponding positions in $B_{C}$ and $B_{D}$ satisfy the condition: $b c_{s t} \times b d_{s t}=0$ and $b c_{t s} \times b d_{t s}=0$, or $b c_{s t} \times b d_{s t}=1$ and $b c_{t s} \times b d_{t s}=1$. Hence, there is no arc between the two alternatives in the outranking graph, which means that they are incomparable. In such cases, we set $B_{s \hat{t}}=1, B_{t \hat{s}}=0$, which indicates that $o p_{s}$ weakly outperforms $o p_{t}$ when $b c_{t s} \times b d_{t \hat{s}}=1$ and $b c_{t \hat{s}} \times b d_{t \hat{s}}=0$.

\subsubsection{The Decision-Making Procedures}

On the basis of the above description, the procedure of the presented expected-HDD-based ELECTRE II method can be given as follows:

Step 1: Obtain the decision matrices $\mathbf{R}^{(\vartheta)}=\left[h_{S_{\phi i j}}^{(\vartheta)}\right]_{m \times n}(\vartheta=1,2, \ldots, g)$ provided by the invited experts. Set the attitude (position) weights of the strong, moderate, and weak DHHFLCS, the DHHFL indifference set, and the strong, moderate, and weak DHHFLDS, represented as $\omega_{c}{ }^{s}, \omega_{c}{ }^{m}, \omega_{c}{ }^{w}, \omega=, \omega_{d}{ }^{s}$, $\omega_{d}^{m}$ and $\omega_{d}{ }^{w}$, respectively.

Step 2: Calculate the DM's weight $w_{\xi}^{(\vartheta)}(\xi=1,2, \ldots, n ; \vartheta=1,2, \ldots, g)$ regarding each attribute by means of the method defined in Section 3.2.

Step 3: Calculate the criteria's weight $\omega_{j}(j=1,2, \ldots, n)$ based on the optimization model and obtain the overall decision matrix $\overline{\mathrm{R}}=\left[\bar{h}_{S_{\phi i j}}\right]_{m \times n}$.

Step 4: Compute the expected value and HDD of the DHHFLEs in the final matrix $\bar{R}$ as

$$
\begin{gathered}
\stackrel{\leftrightarrow}{E} v\left(\bar{h}_{S_{\phi i j}}\right)=\frac{1}{\Delta \bar{h}_{S_{\phi i j}}} \sum_{r=1}^{\Delta \bar{h}_{S_{\phi i j}}} I\left(\zeta_{t_{r}<\delta_{k r}>}\right), \\
h \sigma\left(\bar{h}_{S_{\phi i j}}\right)=\frac{\Delta \bar{h}_{S_{\phi i j}}-1}{2 \varepsilon \tau} \sqrt{\frac{1}{\Delta \bar{h}_{S_{\phi i j}}} \sum_{r=1}^{\Delta \bar{h}_{S_{i j}}}\left(I\left(\zeta_{t_{r}<\delta_{k r}>}\right)-\stackrel{\leftrightarrow}{E}_{v}\left(\bar{h}_{S_{\phi i j}}\right)\right)^{2},}
\end{gathered}
$$

where $\Delta \bar{h}_{S_{\phi i j}}$ is the count of elements in $\bar{h}_{S_{\phi i j}}$

Step 5: Construct the strong, moderate, and weak DHHFLCS by Equations (35)-(37), the strong, moderate, and weak DHHFLDS by Equations (38)-(40), and the DHHFL indifferent set by Equation (41).

Step 6: Calculate the DHHFLCI of any pair of alternatives by Equation (42) to establish the DHHFL concordant matrix $C=\left[C I_{s t}\right]_{m \times m}$.

Calculate the DHHFLDI of any pair of alternatives by Equation (43) to establish the DHHFL discordant matrix $D=\left[D I_{s t}\right]_{m \times m}$.

Step 7: Calculate the critical value as $\bar{C}=\sum_{s=1}^{m} \sum_{t=1}^{m} C I_{s t} / m(m-1)$ and construct the DHHFL concordant Boolean matrix $B_{C}$. 
Calculate the marginal value as $\bar{D}=\sum_{s=1}^{m} \sum_{t=1}^{m} D I_{s t} / m(m-1)$ and construct the DHHFL discordant Boolean matrix $B_{D}$.

Step 8: Establish the comprehensive Boolean matrix $B$ and then depict the outranking graph based on the matrix.

If there is no arc between two vertices in the graph, locate the corresponding symmetrical positions in $B$ where the elements may be not complementary. Then, revise the elements of the same positions in the matrices $B_{C}, B_{D}$, and $B$, and further redraw the outranking graph with the arcs of weak outranking.

To make the decision-making processes of the proposed expected-HDD-based ELECTRE II method clearer, a flowchart is summarized, as shown in Figure 2.

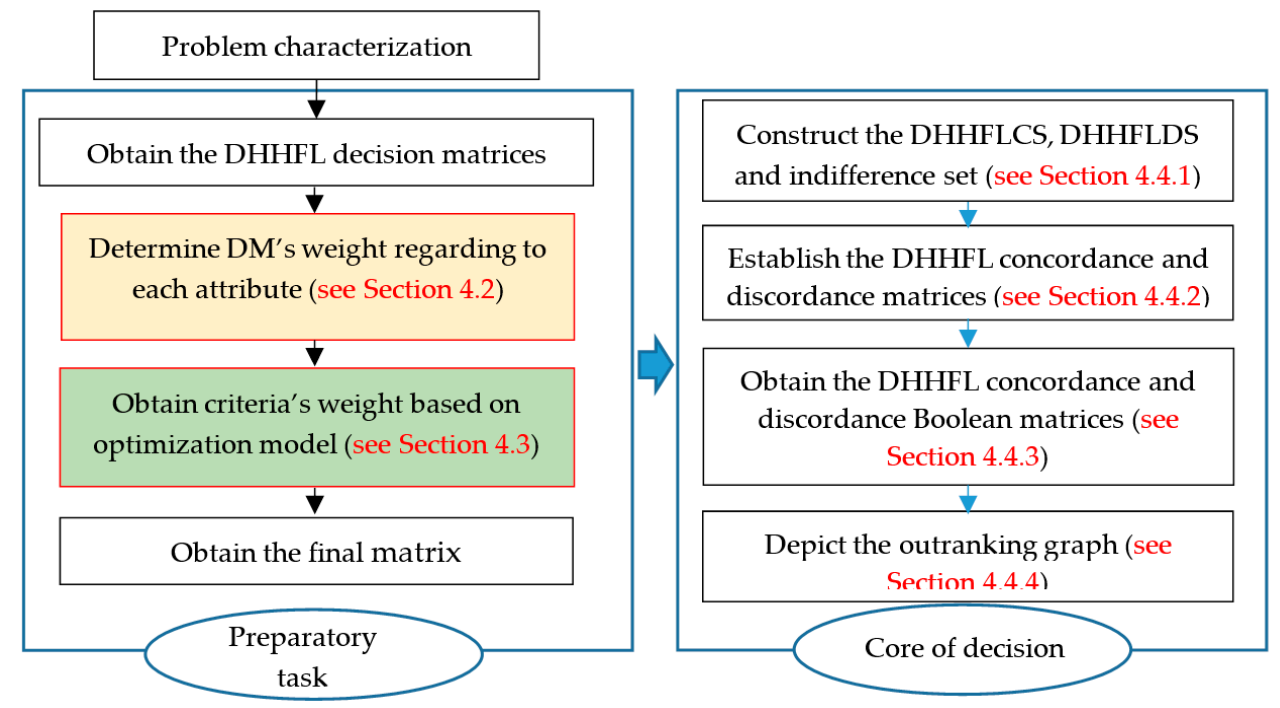

Figure 2. The decision-making process.

The processes of weight-determination in Figure 2 can be redefined as in Figures 3 and 4 , respectively.

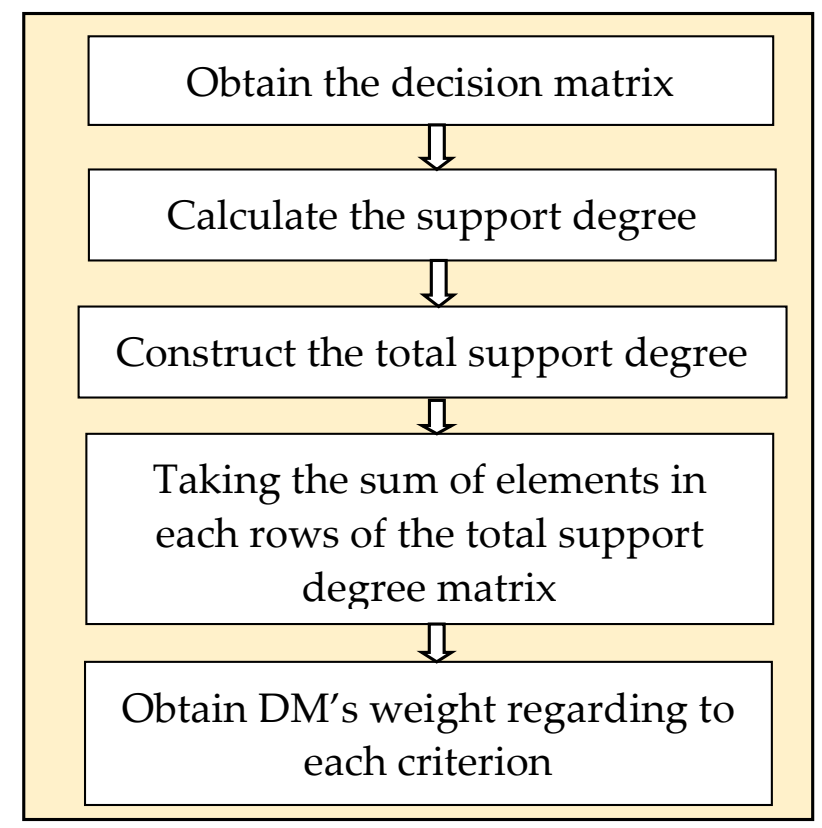

Figure 3. Determine the decision maker's (DM's) weight. 


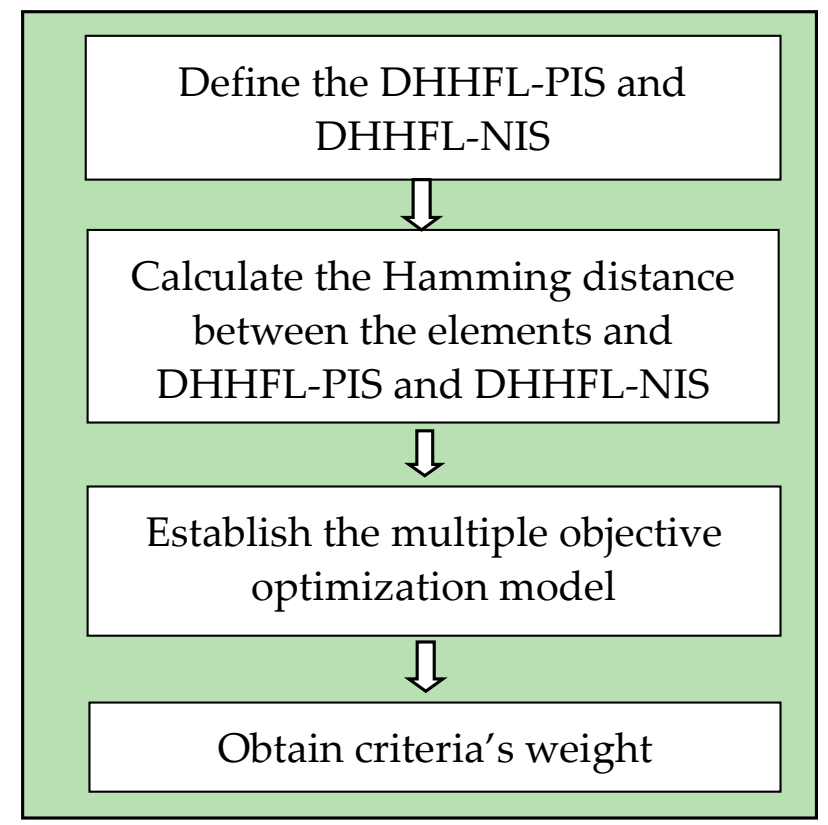

Figure 4. Determine the criteria's weight.

\section{Numerical Example: E-Government Outsourcer Selection}

\subsection{Application Background}

E-government outsourcing (EGO), also known as IT Service Outsourcing in government, is not only a kind of government service outsourcing, but is also an IT outsourcing, which aims to relieve the pressure on government departments and provide more professional service for the public. In the process of government informatization, the government entrusts part or all of the e-government project construction, daily operation, and maintenance, as well as related services, to a professional IT outsourcing service provider to improve the quality of e-government. From the perspective of outsourcing details, the EGO can be divided into the following four categories:

1. Overall outsourcing of IT resources: The outsourcer should provide government departments with a full set of IT system planning, procurement, implementation, operation and maintenance, consulting, and training services.

2. Single IT technology outsourcing: The government will selectively outsource some processes of IT projects, such as network construction, hardware equipment maintenance, and single software development, to the IT service provider.

3. Maintenance outsourcing: The government outsources the maintenance service of the established business system to enjoy the service of professional technical strength.

4. Apart from the abovementioned outsourcing contents, EGO should also include project planning, program design, partner determination, training, and other content.

In short, outsourcing can cover the whole life cycle of e-government projects from planning to implementation, from supervision to the final operation and maintenance. To ensure the service quality and preferably save manpower and material resources, it is vital to effectively pick the outsourcer in the process of EGO, which is an MCDM problem involving multiple experts and criteria. Our proposed method can not only describe the preference of experts more accurately, but can also deal with the non-compensatory problem of criteria, which has significant advantages in solving the MCGDM problem. Thus, in the following, we apply this novel approach to a practical outsourcer selection problem in the process of EGO. 


\subsection{Problem Characteristics}

Due to the aging system, a city government is willing to develop a new public opinion feedback system. To reduce the IT cost and improve service quality as much as possible, the government decided to resort to an IT outsourcing provider to purchase the IT service. After the first round of bidding, five alternate outsourcers stand out: $o p_{1}$ : Digital China Information Service Company Ltd; $o p_{2}$ : AsiaInfo; $o p_{3}$ : DOUPLES; $o p_{4}$ : Neusoft; $o p_{5}$ : ChinasoftInternational. After comprehensively discussing in a departmental meeting, the government picked four experts to evaluate the given alternatives under the five critical criteria shown in Table 2. The expert vectors are represented as $E=\left\{e x_{1}, e x_{2}, e x_{3}, e x_{4}\right\}$ and criteria vectors as $C=\left\{c r_{1}, c r_{2}, c r_{3}, c r_{4}, c r_{5}\right\}$, in which the weights of experts and criteria are completely unknown.

Table 2. Description of critical evaluation index.

\begin{tabular}{cc}
\hline Critical Attribute & Description \\
\hline Economics $\left(c r_{1}\right)$ & This index refers to the developing cost of the outsourcer. \\
Operability $\left(c r_{2}\right)$ & This criterion is based on the available degree of the system. \\
Stability $\left(c r_{3}\right)$ & This criterion depends on the compressive ability and flexibility. \\
Market share $\left(c r_{4}\right)$ & This index can reflect the visibility and reliability of the company. \\
User experience $\left(c r_{5}\right)$ & This refers to the feedback from the previous users. \\
\hline
\end{tabular}

In the process of evaluation, the experts employ the DHHFLE to describe their opinion, where the first-hierarchy LTS is $S=\left\{\zeta_{1}, \zeta_{2}, \zeta_{3}, \zeta_{4}, \zeta_{5}, \zeta_{6}, \zeta_{7}\right\}=\{$ terrible, pretty bad,bad, moderate, well, pretty well, perfect\}, and the corresponding second-hierarchy LTSs are shown in Figure 1.

\subsection{Decision-Making Procedure}

Step 1: Firstly, we preset the position weights of the strong, moderate, and weak DHHFLCS, the DHHFL indifferent set, and the strong, moderate, and weak DHHFLDS as $\widetilde{\omega}=\left(\omega_{c}{ }^{s}, \omega_{c}{ }^{m}, \omega_{c}{ }^{w}, \omega^{=}, \omega_{d}{ }^{s}, \omega_{d}{ }^{m}, \omega_{d}{ }^{w}\right)=(1,0.8,0.6,1,0.9,0.7,0.6)$. The decision matrices of each expert are given respectively as follows:

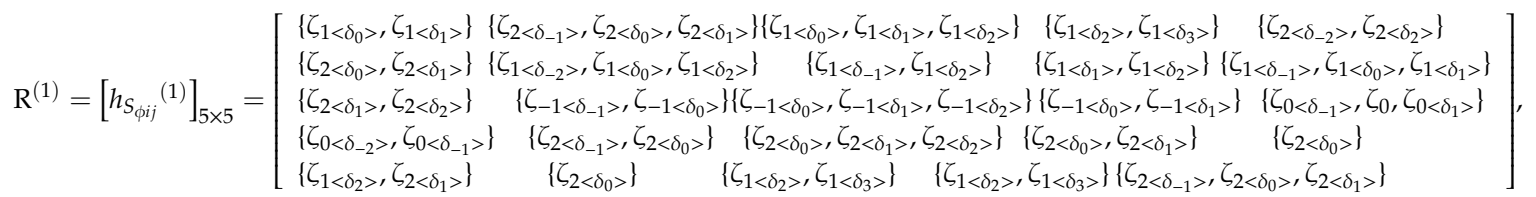

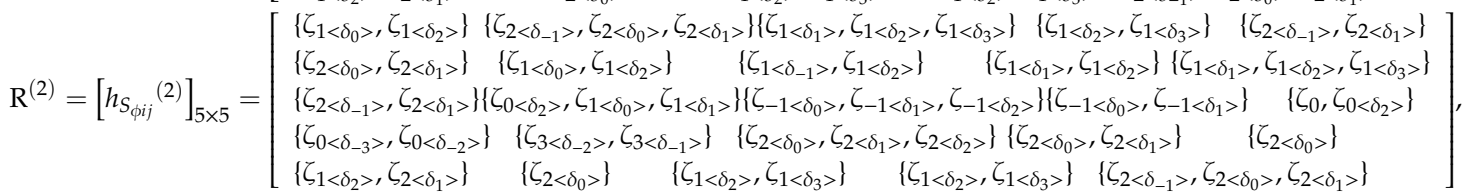

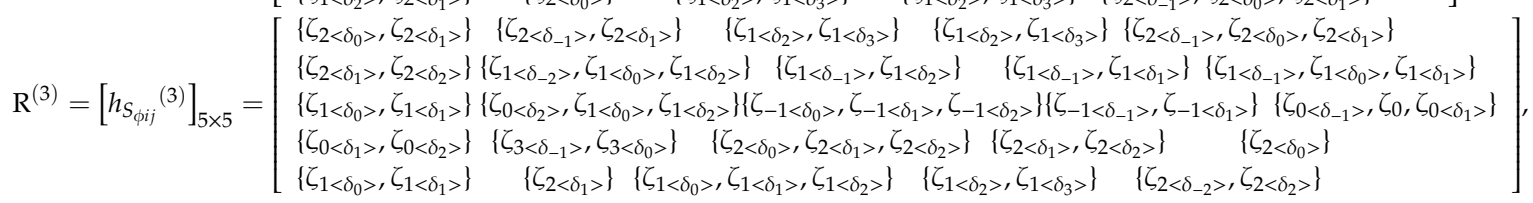

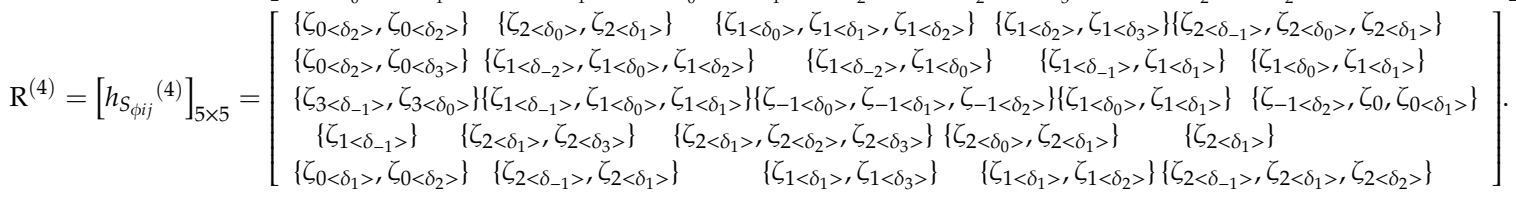

Step 2: Calculate the DM's weight $w_{\xi}^{(\vartheta)}(\xi=1,2,3,4,5 ; \vartheta=1,2,3,4)$ regarding each attribute using the method defined in Section 3.2. 
Step 2.1: Transform the decision matrices $\mathrm{R}^{(\vartheta)}(\vartheta=1,2,3,4)$ into $\mathrm{Q}^{(\xi)}(\xi=1,2,3,4,5)$ as follows:

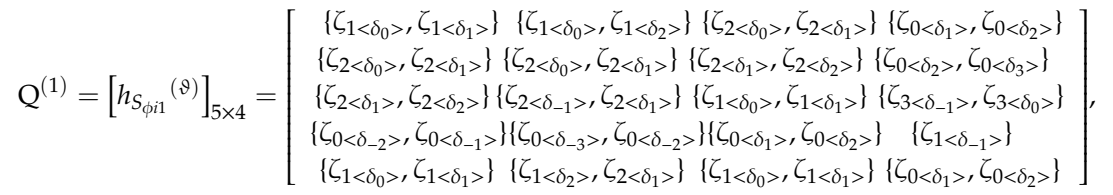

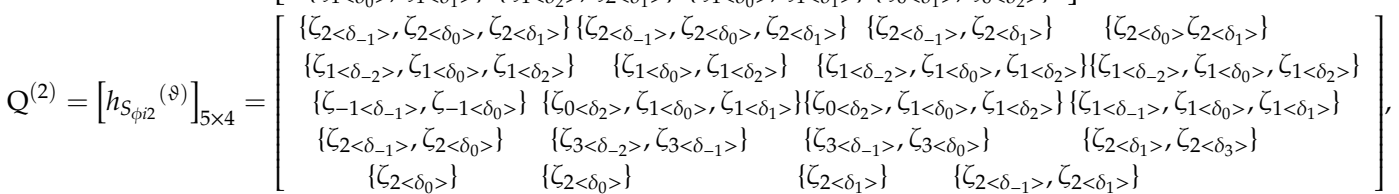

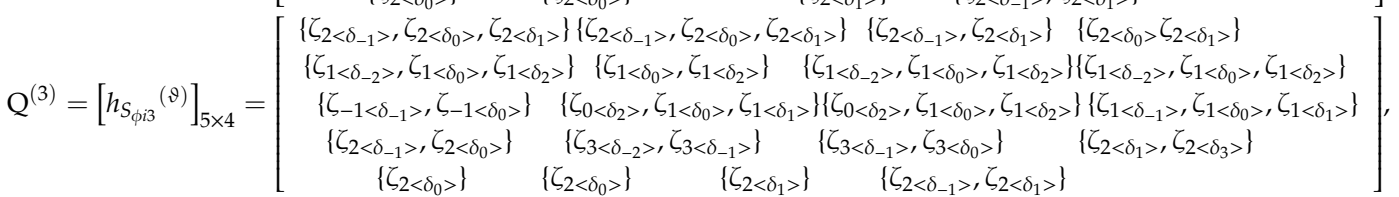

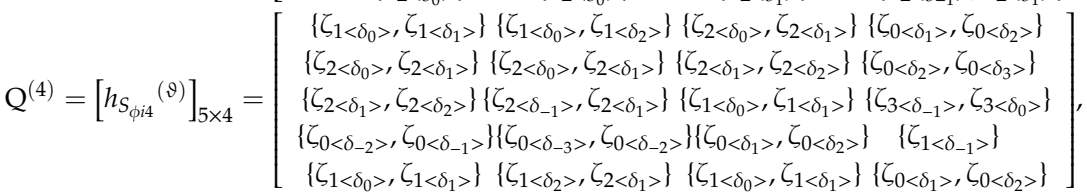

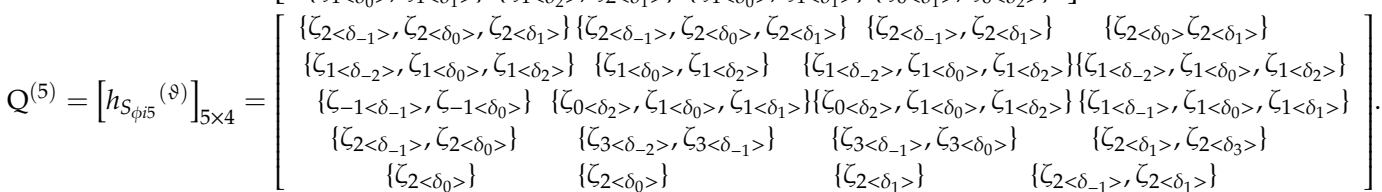

Step 2.2: Obtain the support degrees of $h_{S_{\phi i \xi}}(p)(p=1,2,3,4)$ from $h_{S_{\phi i \xi}}(q)(q=1,2,3,4 ; q \neq p)$ using the Equation (19). For example, the support degrees of $h_{S_{\phi i 1}}{ }^{(1)}$ from $h_{S_{\phi i 1}}(q)(q=2,3,4)$ under the criterion $\mathrm{cr}_{1}$ are shown in Table 3.

Table 3. The support degrees of $h_{S_{\phi i 1}}{ }^{(1)}$ from $h_{S_{\phi i 1}}(q)(q=2,3,4)$.

\begin{tabular}{cccc}
\hline $\boldsymbol{s u p}\left(\boldsymbol{h}_{\boldsymbol{S}_{\boldsymbol{\phi} i 1}}(1), \boldsymbol{h}_{\boldsymbol{S}_{\phi i 1}}(\boldsymbol{q})\right.$ & $\boldsymbol{q})$ & $\boldsymbol{q}=3$ & $\boldsymbol{q}=4$ \\
\hline$i=1$ & 0.9722 & 0.8333 & 0.8889 \\
$i=2$ & 1 & 0.9444 & 0.7778 \\
$i=3$ & 0.9167 & 0.7778 & 0.9444 \\
$i=4$ & 0.9444 & 0.8333 & 0.8056 \\
$i=5$ & 0.8611 & 1 & 0.8889 \\
\hline
\end{tabular}

Step 2.3: Calculate the total support degree $T\left(h_{S_{\phi i \xi}}(p)\right)$ of $h_{S_{\phi i \xi}}(p)(p=1,2,3,4)$ using the following function:

$$
T\left(h_{S_{\phi i \xi}}(p)\right)=\sum_{\substack{q=1 \\ q \neq p}}^{g} \sup \left(h_{S_{\phi i \xi}}(p), h_{S_{\phi i \xi}}(q)\right), i=1,2, \ldots, m ; \xi=1,2, \ldots, n .
$$


The total support degree matrix can be given as below:

$$
\begin{gathered}
T^{(1)}=\left[T\left(h_{S_{\phi i 1}}{ }^{(k)}\right)\right]_{5 \times 4}= \\
\\
o p_{1} \\
o p_{2} \\
o p_{3} \\
o p_{4} \\
o p_{5}
\end{gathered} \quad\left[\begin{array}{rrrrr}
2.69444 & 2.69444 & 2.41667 & 2.47222 \\
2.72222 & 2.72222 & 2.61111 & 2.27778 \\
2.58333 & 2.58333 & 2.30556 & 2.36111 \\
2.58333 & 2.47222 & 2.58333 & 2.52778 \\
2.75 & 2.47222 & 2.75 & 2.52778
\end{array}\right]
$$

Step 2.4: Take the sum of elements in each column of $T^{(\xi)}$ and obtain the overall support degree of each expert regarding the criterion $\mathrm{cr}_{\xi}$. The results are shown as follows:

$$
\begin{aligned}
& l_{1}^{(1)}=13.3889 ; l_{1}{ }^{(2)}=13 ; l_{1}{ }^{(3)}=12.7222 ; l_{1}{ }^{(4)}=12.3333 ; \\
& l_{2}{ }^{(1)}=13.3056 ; l_{2}{ }^{(2)}=14.1389 ; l_{2}{ }^{(3)}=14.0833 ; l_{2}{ }^{(4)}=14.1574 ; \\
& l_{3}(1)=14.6111 ; l_{3}{ }^{(2)}=14.6111 ; l_{3}(3)=14.4444 ; l_{3}{ }^{(4)}=14.3333 ; \\
& l_{4}{ }^{(1)}=14.3611 ; l_{4}{ }^{(2)}=14.3611 ; l_{4}\left({ }^{(3)}=14.1944 ; l_{4}{ }^{(4)}=13.5833 ;\right. \\
& l_{5}{ }^{(1)}=14.5648 ; l_{5}{ }^{(2)}=14.4609 ; l_{5}{ }^{(3)}=14.6019 ; l_{5}{ }^{(4)}=14.4907 ;
\end{aligned}
$$

Step 2.5: Obtain each DM's weight regarding to the criterion $\mathrm{cr}_{\xi}$, and the consequences are presented below.

$$
\begin{aligned}
& w_{1}^{(1)}=0.26026 ; w_{1}^{(2)}=0.2527 ; w_{1}^{(3)}=0.2473 ; w_{1}^{(4)}=0.23974 ; \\
& w_{2}{ }^{(1)}=0.23894 ; w_{2}{ }^{(2)}=0.25391 ; w_{2}{ }^{(3)}=0.25291 ; w_{2}{ }^{(4)}=0.25424 ; \\
& w_{3}{ }^{(1)}=0.25192 ; w_{3}{ }^{(2)}=0.25192 ; w_{3}{ }^{(3)}=0.24904 ; w_{3}{ }^{(4)}=0.24713 \text {; } \\
& w_{4}{ }^{(1)}=0.25418 ; w_{4}{ }^{(2)}=0.25418 ; w_{4}{ }^{(3)}=0.25123 ; w_{4}{ }^{(4)}=0.24041 ; \\
& w_{5}{ }^{(1)}=0.25104 ; w_{5}^{(2)}=0.24753 ; w_{5}{ }^{(3)}=0.25168 ; w_{5}{ }^{(4)}=0.24976 \text {. }
\end{aligned}
$$

Step 3: Calculate the criterion's weight $\omega_{j}(j=1,2,3,4,5)$ based on the optimization model and obtain the overall decision matrix $\mathrm{R}=\left[h_{S_{\phi i j}}\right]_{m \times n}$.

After getting each expert's weight regarding each attribute, we can obtain the overall weighted matrix R:

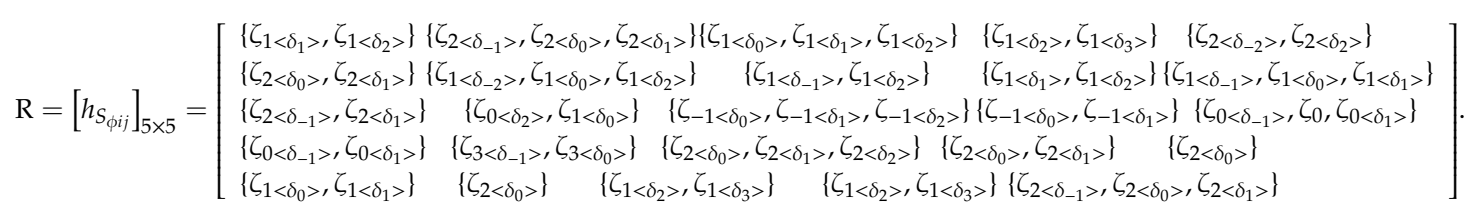


Step 3.1: Obtain the DHHFL positive ideal solution (DHHFL-PIS) and the DHHFL negative ideal solution (DHHFL-NIS) by Equations (25) and (26), respectively.

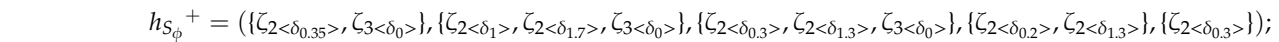

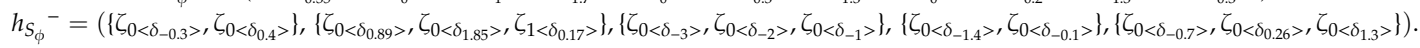

Step 3.2: Calculate the Hamming distance $h d\left(h_{S_{\phi i j}} h_{S_{\phi j}}{ }^{+}\right)(i=1,2, \ldots, m ; j=1,2, \ldots, n)$. What follows are the results of the calculation (Table 4).

Table 4. The distance between the evaluation and the double hierarchy hesitant fuzzy linguistic positive ideal solution (DHHFL-PIS).

\begin{tabular}{cccccc}
\hline $\boldsymbol{h d}\left(\boldsymbol{h}_{\boldsymbol{S}_{\phi i j}} \boldsymbol{h}_{\boldsymbol{S}_{\phi j}}{ }^{+}\right)$ & $j=1$ & $j=2$ & $j=3$ & $j=4$ & $j=5$ \\
\hline$i=1$ & 0.1903 & 0.099 & 0.1587 & 0.0725 & 0.0526 \\
$i=2$ & 0.0797 & 0.258 & 0.2425 & 0.166 & 0.1441 \\
$i=3$ & 0 & 0.3307 & 0.5296 & 0.4188 & 0.3350 \\
$i=4$ & 0.4247 & 0 & 0 & 0 & 0 \\
$i=5$ & 0.2071 & 0.0894 & 0.138 & 0.0846 & 0.0522 \\
\hline
\end{tabular}

Step 3.3: Calculate the Hamming distance $h d\left(h_{S_{\phi i j}} h_{S_{\phi j}}{ }^{-}\right)(i=1,2, \ldots, m ; j=1,2, \ldots, n)$. The results are shown in Table 5.

Table 5. The distance between the evaluation and the DHHFL negative ideal solution (DHHFL-NIS).

\begin{tabular}{cccccc}
\hline $\boldsymbol{h} \boldsymbol{d}\left(\boldsymbol{h}_{\boldsymbol{S}_{\phi i j}} \boldsymbol{h}_{\boldsymbol{S}_{\phi j}}{ }^{-}\right)$ & $j=1$ & $j=2$ & $j=3$ & $j=4$ & $j=5$ \\
\hline$i=1$ & 0.2343 & 0.2317 & 0.3711 & 0.3462 & 0.3206 \\
$i=2$ & 0.345 & 0.0727 & 0.2871 & 0.2528 & 0.1909 \\
$i=3$ & 0.4247 & 0 & 0 & 0 & 0 \\
$i=4$ & 0 & 0.3307 & 0.5296 & 0.4188 & 0.3350 \\
$i=5$ & 0.2176 & 0.2413 & 0.3916 & 0.3342 & 0.3309 \\
\hline
\end{tabular}

Step 3.4: Establish the optimization model and obtain the weight vector of the criteria.

$$
(M)\left\{\begin{array}{l}
\max H=\sum_{i=1}^{m} \sum_{j=1}^{n}\left(\left(h d\left(h_{S_{\phi i j}} h_{S_{\phi j}}{ }^{+}\right)+h d\left(h_{S_{\phi i j}}, h_{S_{\phi j}}{ }^{-}\right)\right) \times \omega_{j}\right) \\
\text { s.t. } \sum_{j=1}^{n} \omega_{j}^{2}=1, \omega_{j} \in N, j=1,2, \ldots, n
\end{array} .\right.
$$

Solving the model M, we can obtain the weight of each criterion as follows:

$$
\omega_{1}{ }^{\prime}=0.4327 ; \omega_{2}{ }^{\prime}=0.3104 ; \omega_{3}{ }^{\prime}=0.5595 ; \omega_{4}{ }^{\prime}=0.4789 ; \omega_{5}{ }^{\prime}=0.4171 .
$$

Using Equation (21) to standardize them, we can obtain:

$$
\omega_{1}=0.1968 ; \omega_{2}=0.1412 ; \omega_{3}=0.2545 ; \omega_{4}=0.2178 ; \omega_{5}=0.1897 .
$$

Then, we can get the following final decision matrix:

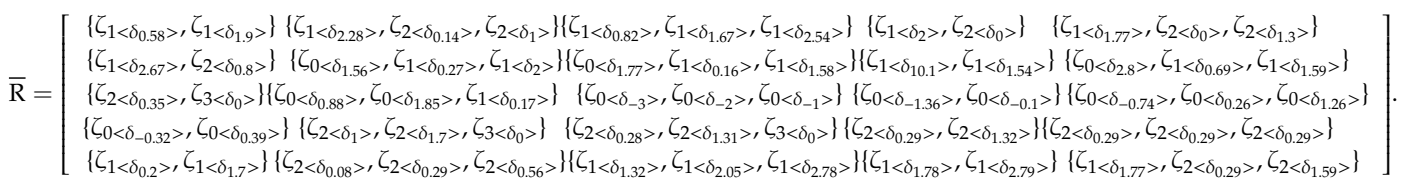

Step 4: Compute the expected value and HDD of the DHHFLEs in the final matrix $\bar{R}$, which are shown in Tables 6 and 7, respectively. 
Table 6. The expected value $\stackrel{\leftrightarrow}{E} v\left(\bar{h}_{S_{\phi i j}}\right)$

\begin{tabular}{cccccc}
\hline$\stackrel{\leftrightarrow}{\boldsymbol{E}} \boldsymbol{v}\left(\boldsymbol{h}_{\boldsymbol{S}_{\phi i j}}\right)$ & $j=1$ & $j=2$ & $j=3$ & $j=4$ & $j=5$ \\
\hline$i=1$ & 0.2318 & 0.2316 & 0.3062 & 0.3013 & 0.2969 \\
$i=2$ & 0.3110 & 0.1526 & 0.2522 & 0.2388 & 0.2081 \\
$i=3$ & 0.6571 & 0.1253 & 0.1182 & 0.1257 & 0.1286 \\
$i=4$ & 0.1282 & 0.5270 & 0.6116 & 0.3707 & 0.3017 \\
$i=5$ & 0.2227 & 0.2357 & 0.3216 & 0.2919 & 0.3083 \\
\hline
\end{tabular}

Table 7. The hesitant deviation degree $h \sigma\left(\bar{h}_{S_{\phi i j}}\right)$.

\begin{tabular}{cccccc}
\hline $\boldsymbol{h} \sigma\left(\boldsymbol{h}_{\boldsymbol{S}_{\boldsymbol{i j}}}\right)$ & $j=1$ & $j=2$ & $j=3$ & $j=4$ & $j=5$ \\
\hline$i=1$ & 0.0019 & 0.0031 & 0.0032 & 0.0012 & 0.0056 \\
$i=2$ & 0.0016 & 0.0034 & 0.0042 & 0.0013 & 0.0023 \\
$i=3$ & 0.0191 & 0.0019 & 0.0019 & 0.0007 & 0.0017 \\
$i=4$ & 0.0004 & 0.0372 & 0.0307 & 0.0018 & 0 \\
$i=5$ & 0.0013 & 0.0009 & 0.0029 & 0.0012 & 0.0065 \\
\hline
\end{tabular}

Step 5: Construct the matrices of strong, moderate, and weak DHHFLCS, which are represented as $J c^{s}, J c^{m}$, and $J c^{w}$, and shown as

$$
\begin{aligned}
& J c^{s}=\left[J c_{p q}{ }^{s}\right]_{5 \times 5}=\left[\begin{array}{ccccc}
\backslash & \{2,3,4\} & - & - & \{1\} \\
- & \backslash & - & - & - \\
- & - & \backslash & - & - \\
\{5\} & \{5\} & \{5\} & \backslash & - \\
\{2,3\} & \{2,3,4\} & \{2\} & - & \backslash
\end{array}\right], \\
& J c^{m}=\left[J c_{p q}{ }^{m}\right]_{5 \times 5}=\left[\begin{array}{cccccc}
\backslash & \{5\} & \{2,3,4,5\} & \{1\} & \{4\} \\
\{1\} & \backslash & \{2,3,4,5\} & \{1\} & \{1\} \\
\{1\} & \{1\} & \backslash & \{1\} & \{1\} \\
\{2,3,4\} & \{2,3,4\} & \{2,3,4\} & \backslash & \{2,3,4\} \\
\{5\} & \{5\} & \{3,4,5\} & \{1,5\} & \backslash
\end{array}\right], \\
& J c^{w}=\left[J c_{p q}{ }^{w}\right]_{5 \times 5}=\left[\begin{array}{ccccc}
\backslash & - & - & - \\
- & \backslash & - & - & - \\
- & - & \backslash & - & - \\
- & - & - & \backslash & - \\
- & - & - & - & \backslash
\end{array}\right] .
\end{aligned}
$$


Establish the matrices of strong, moderate, and weak DHHFLDS, denoted as $J d^{s}, J d^{m}$, and $J d^{w}$, respectively.

$$
\begin{aligned}
J d^{s}=\left[J d_{p q^{s}}\right]_{5 \times 5}= & {\left[\begin{array}{ccccc}
\backslash & - & - & \{5\} & \{2,3\} \\
\{2,3,4\} & \backslash & - & \{5\} & \{2,3,4\} \\
- & - & \backslash & \{5\} & \{2\} \\
- & - & - & \backslash & - \\
\{1\} & - & - & - & \backslash
\end{array}\right], } \\
J d^{m}=\left[J d_{p q}{ }^{m}\right]_{5 \times 5}= & {\left[\begin{array}{cccccc}
\backslash & & \{1\} & \{1\} & \{2,3,4\} & \{5\} \\
\{2,3,4\} & \backslash & - & \{5\} & \{2,3,4\} \\
\{2,3,4,5\} & \{2,3,4,5\} & \backslash & \{2,3,4\} & \{3,4,5\} \\
\{1\} & & \{1\} & \{1\} & \backslash & \{1,5\} \\
\{4\} & & \{1\} & \{1\} & \{2,3,4\} & \backslash
\end{array}\right], } \\
J d^{w}=\left[J d_{p q}{ }^{w}\right]_{5 \times 5}= & {\left[\begin{array}{ccccc}
\backslash & - & - & - \\
- & \backslash & - & - & - \\
- & - & \backslash & - & - \\
- & - & - & \backslash & - \\
- & - & - & - & \backslash
\end{array}\right] . }
\end{aligned}
$$

Similarly, the DHHFL indifferent set is shown as

$$
J^{=}=\left[J_{p q}=\right]_{5 \times 5}=\left[\begin{array}{ccccc}
1 & - & - & - & - \\
- & 1 & - & - & - \\
- & - & 1 & - & - \\
- & - & - & 1 & - \\
- & - & - & - & 1
\end{array}\right]
$$

Step 6: Calculate the DHHFLCI of any pair of alternatives and establish the DHHFL concordant matrix $C=\left[C I_{s t}\right]_{m \times m}$ as follows:

$$
C=\left[C I_{s t}\right]_{m \times m}=\left[\begin{array}{ccccc}
- & 0.7652 & 0.6426 & 0.1575 & 0.3711 \\
0.1575 & - & 0.6426 & 0.1575 & 0.1575 \\
0.1575 & 0.1575 & - & 0.1575 & 0.1575 \\
0.6805 & 0.6805 & 0.6805 & - & 0.4908 \\
0.5474 & 0.7652 & 0.6708 & 0.3092 & -
\end{array}\right]
$$

Calculate the DHHFLDI of any pair of alternatives to establish the DHHFL discordant matrix $D=\left[D I_{s t}\right]_{m \times m}$ below.

$$
D=\left[D I_{s t}\right]_{m \times m}=\left[\begin{array}{ccccc}
- & 0.1228 & 0.1378 & 0.1781 & 0.1961 \\
0.1392 & - & 0.1378 & 0.1710 & 0.1587 \\
0.0787 & 0.0689 & - & 0.1662 & 0.0834 \\
0.0467 & 0.0673 & 0.1378 & 0.0446 & 0.0446 \\
0.0901 & 0.1215 & 0.1378 & 0.1772 & -
\end{array}\right]
$$

Step 7: Compute the critical value, we can obtain

$$
\bar{C}=\sum_{s=1}^{5} \sum_{t=1}^{5} C I_{s t} / m(m-1)=0.4253 .
$$


Then, further construct the DHHFL concordant Boolean matrix $B_{C}$ as:

$$
B_{C}=\left[b c_{s t}\right]_{5 \times 5}=\left[\begin{array}{ccccc}
- & 1 & 1 & 0 & 0 \\
0 & - & 1 & 0 & 0 \\
0 & 0 & - & 0 & 0 \\
1 & 1 & 1 & - & 1 \\
1 & 1 & 1 & 0 & -
\end{array}\right],
$$

Calculate the marginal value as

$$
\bar{D}=\sum_{s=1}^{m} \sum_{t=1}^{m} D I_{s t} / m(m-1)=0.1253 .
$$

Further construct the DHHFL discordant Boolean matrix $B_{D}$ as:

$$
B_{D}=\left[b d_{s t}\right]_{5 \times 5}=\left[\begin{array}{ccccc}
- & 1 & 0 & 0 & 0 \\
0 & - & 0 & 0 & 0 \\
1 & 1 & - & 0 & 1 \\
1 & 1 & 0 & - & 1 \\
1 & 1 & 0 & 0 & -
\end{array}\right] .
$$

Step 8: Establish the comprehensive Boolean matrix $B$ as:

$$
B=\left[b c_{s t} \times b d_{s t}\right]_{5 \times 5}=\left[\begin{array}{ccccc}
- & 1 & 0 & 0 & 0 \\
0 & - & 0 & 0 & 0 \\
0 & 0 & - & 0 & 0 \\
1 & 1 & 0 & - & 1 \\
1 & 1 & 0 & 0 & -
\end{array}\right]
$$

Based on the matrix, the outranking graph can be depicted as below:

As shown in Figure 5, there have no arcs between $o p_{3}$ and other options, which means that the corresponding elements in symmetrical positions of $B$ are not complementary. Thus, in the following, we are going to locate the positions and sign them with "'n'; then, we can have

$$
B=\left[b c_{s t} \times b d_{s t}\right]_{5 \times 5}=\left[\begin{array}{ccccc}
- & 1 & \hat{0} & 0 & 0 \\
0 & - & \hat{0} & 0 & 0 \\
\hat{0} & \hat{0} & - & \hat{0} & \hat{0} \\
1 & 1 & \hat{0} & - & 1 \\
1 & 1 & \hat{0} & 0 & -
\end{array}\right]
$$

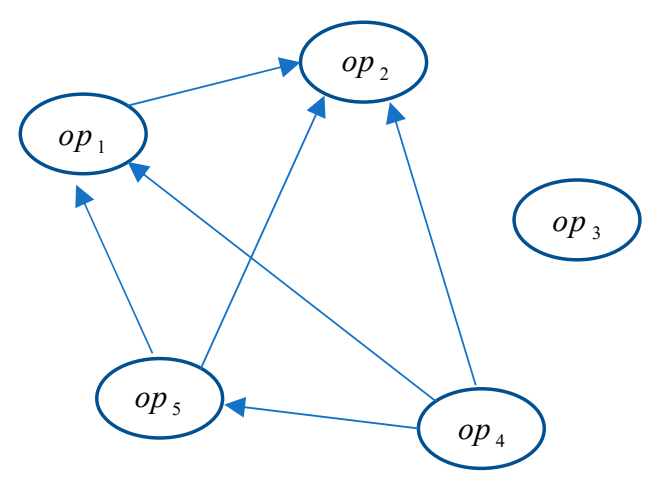

Figure 5. The strongly outranking graph. 
Then, the corresponding positions in matrices $B_{C}$ and $B_{D}$ are rebuilt as:

$$
B_{C}=\left[b c_{s t}\right]_{5 \times 5}=\left[\begin{array}{ccccc}
- & 1 & 1 & 0 & 0 \\
0 & - & 1 & 0 & 0 \\
0 & 0 & - & 0 & 0 \\
1 & 1 & 1 & - & 1 \\
1 & 1 & 1 & 0 & -
\end{array}\right], B_{D}=\left[b d_{s t}\right]_{5 \times 5}=\left[\begin{array}{ccccc}
- & 1 & \hat{1} & 0 & 0 \\
0 & - & \hat{1} & 0 & 0 \\
1 & 1 & - & 0 & 1 \\
1 & 1 & \hat{1} & - & 1 \\
1 & 1 & \hat{1} & 0 & -
\end{array}\right]
$$

Accordingly, the updated $B$ can be given as:

$$
B=\left[b c_{s t} \times b d_{s t}\right]_{5 \times 5}=\left[\begin{array}{ccccc}
- & 1 & 1 & 0 & 0 \\
0 & - & 1 & 0 & 0 \\
0 & 0 & - & 0 & 0 \\
1 & 1 & 1 & - & 1 \\
1 & 1 & 1 & 0 & -
\end{array}\right]
$$

Then, the outranking graph is redrawn and shown in Figure 6.

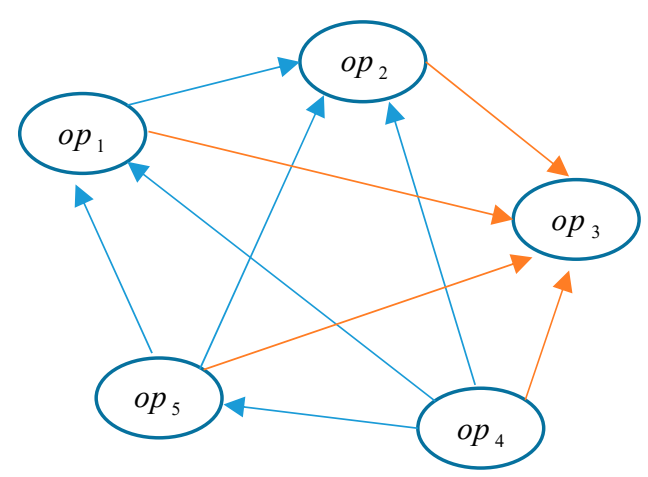

Figure 6. The final outranking graph.

Step 8: Rank the alternatives according to the final outranking graph:

$$
o p_{4}>o p_{5}>o p_{1}>o p_{2}>o p_{3}
$$

\section{Comparative Analysis}

\subsection{Effectiveness Analysis}

Considering that our weight-determination method for experts is motivated by the PA operator, thus, in the following, we extend the method based on the weighted PA operator [46] into the DHHFL environment to deal with the problem of IT outsourcer selection provided in the Section 4.2. The double hierarchy hesitant fuzzy linguistic power average (DHHFLPA) operator is firstly defined as follows:

Definition 5. Given a collection of DHHFLEs $h_{S_{\phi c}}(c=1,2, \ldots, n)$, the definition of the DHHFLPA operator is given as follows:

$$
\operatorname{DHHFLPA}\left(h_{S_{\phi 1}}, h_{S_{\phi 2}}, \ldots, h_{S_{\phi n}}\right)=\sum_{\alpha=1}^{n} \frac{1+T\left(h_{S_{\phi \alpha}}\right)}{\sum_{\ell=1}^{n} 1+T\left(h_{S_{\phi \ell}}\right)} h_{S_{\phi \alpha^{\prime}}}
$$

where $T\left(h_{S_{\phi c}}\right)=\sum_{z=1}^{n} \operatorname{Sup}\left(h_{S_{\phi c}}, h_{S_{\phi z}}\right)$, with $\operatorname{Sup}\left(h_{S_{\phi c}}, h_{S_{\phi z}}\right)$ being the support level of $h_{S_{\phi c}}$ from $h_{S_{\phi z^{\prime}}}$ which

$$
z \neq c
$$

satisfies three characteristics: 
1. $\operatorname{Sup}\left(h_{S_{\phi c}}, h_{S_{\phi z}}\right) \in[0,1]$;

2. $\operatorname{Sup}\left(h_{S_{\phi c}}, h_{S_{\phi z}}\right)=\operatorname{Sup}\left(h_{S_{\phi z}}, h_{S_{\phi c}}\right)$;

3. $\operatorname{Sup}\left(h_{S_{\phi c}}, h_{S_{\phi z}}\right) \geq \operatorname{Sup}\left(\bar{h}_{S_{\phi c}}, \bar{h}_{S_{\phi z}}\right)$, if $d\left(h_{S_{\phi c}}, h_{S_{\phi z}}\right) \leq d\left(\bar{h}_{S_{\phi c}} \bar{h}_{S_{\phi z}}\right)$, where $d\left(h_{S_{\phi c}}, h_{S_{\phi z}}\right)$ is the distance between $h_{S_{\phi c}}$ and $h_{S_{\phi z}}$.

Similarly, the double hierarchy hesitant fuzzy linguistic weighted power average (DHHFLWPA) operator can be defined as:

Definition 6. Given a collection of DHHFLEs $h_{S_{\phi c}}(c=1,2, \ldots, n)$, the definition of the DHHFLWPA operator is given as follows:

$$
\operatorname{DHHFLWPA}\left(h_{S_{\phi 1}}, h_{S_{\phi 2}}, \ldots, h_{S_{\phi n}}\right)=\sum_{\alpha=1}^{n} \frac{\omega_{\alpha}\left(1+T\left(h_{S_{\phi \alpha}}\right)\right.}{\sum_{\ell=1}^{n} \omega_{\ell}\left(1+T\left(h_{S_{\phi \ell}}\right)\right.} h_{S_{\phi \alpha}},
$$

where $T\left(h_{S_{\phi c}}\right)=\sum_{z=1}^{n} \operatorname{Sup}\left(h_{S_{\phi c}}, h_{S_{\phi z}}\right)$, with $\operatorname{Sup}\left(h_{S_{\phi c}}, h_{S_{\phi z}}\right)$ being the support level of $h_{S_{\phi c}}$ from $h_{S_{\phi z}}$. In $z \neq c$

this paper, we use Equation (19) to calculate the support degrees of DHHFLEs.

For convenience, we still use the weight-determination method given in this paper to determine the weights of experts and criteria. Then, referring to the method proposed by Wei et al., the rest of the steps are shown as follows.

Using the DHHFLWPA operator to aggregate the elements in the matrix R, we derive the overall evaluation value of each alternative as:

$$
\begin{aligned}
& \bar{h}_{\delta_{\phi 1}}=\left\{\zeta_{1<\delta_{1.467}>}, \zeta_{1<\delta_{2.2995}>}, \zeta_{2<\delta_{0.2048}>}\right\} ; \bar{h}_{S_{\phi 2}}=\left\{\zeta_{1<\delta_{0.1621}>}>\zeta_{1<\delta_{1.151}>}, \zeta_{\left.1<\delta_{2.1642}\right\rangle}\right\} ; \\
& \bar{h}_{S_{\phi 3}}=\left\{\zeta_{0<\delta_{0.764}>}, \zeta_{0<\delta_{2.181}>}>\zeta_{3<\delta_{0}>}\right\rangle ; \bar{h}_{S_{\phi 4}}=\left\{\zeta_{1<\delta_{2.85}>}>\zeta_{2<\delta_{0.542}>}, \zeta_{3<\delta_{0}>}\right\rangle ; \bar{h}_{\phi_{\phi 5}}=\left\{\zeta_{1<\delta_{1.625}>}, \zeta_{1<\delta_{2.391}>}, \zeta_{2<\delta_{0.198}>}\right\} \text {. }
\end{aligned}
$$

We calculate the expected value and the HDD of the overall evaluation $\bar{h}_{S_{\phi i}}(i=1,2,3,4,5)$, and rank the alternatives based on the proposed comparison. The result is shown in Table 8.

Table 8. The ranking result obtained by the double hierarchy hesitant fuzzy linguistic weighted power average (DHHFLWPA) operator.

\begin{tabular}{ccc}
\hline Method & $\left.\boldsymbol{h \sigma}\left(\overline{\boldsymbol{h}}_{S_{\phi i}}\right), \stackrel{\leftrightarrow}{\boldsymbol{E} v} \overline{\boldsymbol{h}}_{S_{\phi i}}\right)$ & Ranking \\
\hline$h \sigma\left(\bar{h}_{S_{\phi 1}}\right)=0.7958 ; \stackrel{\leftrightarrow}{E} v\left(\bar{h}_{S_{\phi 1}}\right)=0.0043$ \\
$h \sigma\left(\bar{h}_{S_{\phi 2}}\right)=0.7311 ; \stackrel{\leftrightarrow}{E} v\left(\bar{h}_{S_{\phi 2}}\right)=0.005$ \\
$h \sigma\left(\bar{h}_{S_{\phi 3}}\right)=0.7212 ; \stackrel{\leftrightarrow}{E} v\left(\bar{h}_{S_{\phi 3}}\right)=0.0221 \quad o_{4}>o p_{5}>o p_{1}>o p_{2}>o p_{3}$ \\
$h \sigma\left(\bar{h}_{S_{\phi 4}}\right)=0.8962 ; \stackrel{\leftrightarrow}{E} v\left(\bar{h}_{S_{\phi 4}}\right)=0.0083$ \\
$h \sigma\left(\bar{h}_{S_{\phi 5}}\right)=0.8003 ; \stackrel{\leftrightarrow}{E} v\left(\bar{h}_{S_{\phi 5}}\right)=0.004$
\end{tabular}

From Table 8, we can find that the ranking result obtained by the DHHFLWPA operator is the same as that obtained by the expected-HDD-ELECTRE II method. Therefore, the improved ELECTRE II method is proven to be effective and reasonable.

\subsection{Superiority Analysis}

In this section, under the DHHFL environment, two pairs of comparisons are provided to elucidate the advancement of the proposed expected-HDD-based ELECTRE II method, which are among the 
traditional ELECTRE II method [34], the positive and negative ideal-based ELECTRE II method presented by Liao et al. [42], and our newly presented method, respectively.

\subsubsection{Comparison with the Traditional ELECTRE II Method}

In the following, the traditional ELECTRE II method is applied to address the PSO selection issue provided in Section 4.2. Here, based on DHHFLCI and DHHFLDI, the strong outranking relation and weak ranking relation are defined as $O^{S}$ and $O^{W}$, respectively.

Let $c^{-}, c^{\circ}$ and $c^{+}$represent three concordance degrees with $0 \leq c^{-}<c^{\circ}<c^{+} \leq 1$, and $d^{-}$and $d^{+}$ denote the discordance degrees satisfying $0 \leq d^{-}<d^{+} \leq 1$. Then, the strong outranking relations $o p_{x} O^{S} o p_{y}$ and $o p_{x} \mathrm{O}^{W} o p_{y}$ can be respectively given as follows:

(1) $o p_{x} \mathrm{O}^{S} o p_{y}$ can be given as long as one of the following conditions is satisfied:

$$
o p_{x} O^{S} o p_{y} \rightarrow\left\{\begin{array} { l } 
{ C I _ { x y } > C I _ { y x } } \\
{ C I _ { x y } \geq c ^ { + } } \\
{ D I _ { x y } \leq d ^ { + } }
\end{array} \text { or } \left\{\begin{array}{l}
C I_{x y}>C I_{y x} \\
C I_{x y} \geq c^{0} \\
D I_{x y} \leq d^{-}
\end{array} ;\right.\right.
$$

(2) $o p_{x} \mathrm{O}^{W} o p_{y}$ can be established when the following conditions are satisfied:

$$
o p_{x} O^{W} o p_{y} \rightarrow\left\{\begin{array}{l}
C I_{x y}>C I_{y x} \\
C I_{x y} \geq c^{-} \\
D I_{x y} \leq d^{+}
\end{array} .\right.
$$

Here, we set the concordance degrees $\left(c^{-}, c^{\circ}, c^{+}\right)=(0.7,0.6,0.5)$ and the discordance degrees $\left(d^{-}, d^{+}\right)=(0.15,0.3)$. Then, the outranking relation can be established in Table 9.

Table 9. The outranking relation between the alternate options.

\begin{tabular}{cccccc}
\hline Alternatives & $\boldsymbol{o} \boldsymbol{p}_{1}$ & $\boldsymbol{o} \boldsymbol{p}_{2}$ & $\boldsymbol{o} \boldsymbol{p}_{3}$ & $\boldsymbol{o} \boldsymbol{p}_{4}$ & $\boldsymbol{o} \boldsymbol{p}_{5}$ \\
\hline$o p_{1}$ & - & $O^{S}$ & $O^{W}$ & - & - \\
$o p_{2}$ & - & - & $O^{W}$ & - & - \\
$o p_{3}$ & - & - & - & - & - \\
$o p_{4}$ & $O^{S}$ & $O^{S}$ & $O^{W}$ & - & - \\
$o p_{5}$ & $O^{W}$ & $O^{S}$ & $O^{W}$ & - & - \\
\hline
\end{tabular}

In accord with the decision processes of the classical ELECTRE II, the strong and weak outranking graphs can be drawn as in Figures 7 and 8, respectively.

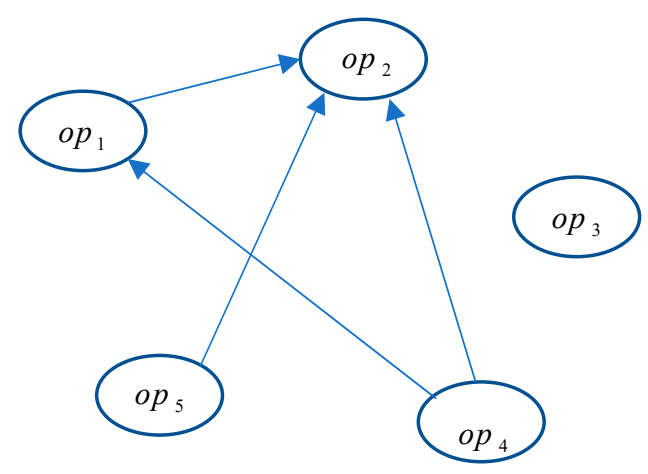

Figure 7. The strong outranking graph. 


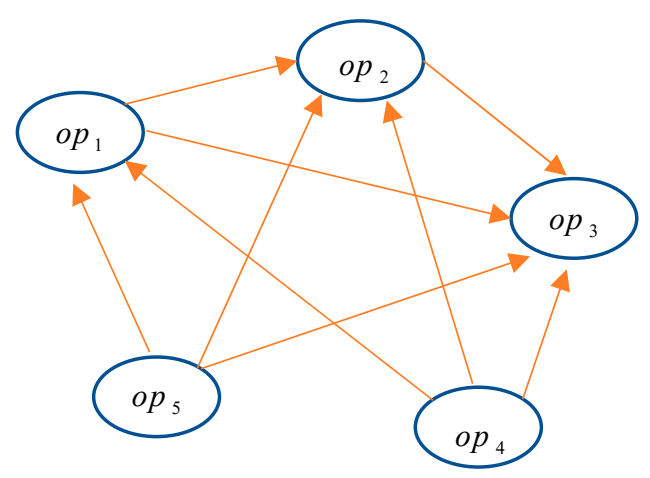

Figure 8. The weak outranking graph.

According to the ranking algorithm, the calculated positive ranking $r^{\prime}(o p)$ and reverse ranking $r^{\prime \prime}(o p)$ are shown in Table 10.

Table 10. The values of $r^{\prime}(o p)$ and $r^{\prime \prime}(o p)$.

\begin{tabular}{cccccc}
\hline Options & $\boldsymbol{o} \boldsymbol{p}_{1}$ & $\boldsymbol{o p _ { 2 }}$ & $\boldsymbol{o p _ { 3 }}$ & $\boldsymbol{o} \boldsymbol{p}_{4}$ & $\boldsymbol{o p _ { 5 }}$ \\
\hline$r^{\prime}(o p)$ & 2 & 3 & 4 & 1 & 1 \\
$r^{\prime \prime}(o p)$ & 3 & 2 & 1 & 4 & 4 \\
\hline
\end{tabular}

Averaging the values of $r^{\prime}(o p)$ and $r^{\prime \prime}(o p)$, we can obtain the final ranking result:

$$
o p_{4} \sim o p_{5}>o p_{1}>o p_{2}>o p_{3} .
$$

Focusing on the ranking result, we can find that $o p_{4}$ is indifferent to $o p_{5}$, while $o p_{4}$ is superior to $o p_{5}$ in the results of our proposed method, which indicates that the orthodox ELECTRE II method cannot accurately identify the subtle differences between the two schemes. Two reasons can account for this difference: (1) Influenced by the levels of concordance and discordance, the classical ELECTRE II method occupies a considerable subjectivity and is not insensitive to the subtle nuances between evaluations; (2) the newly proposed method takes the average of the overall $\mathrm{CI}$ as a critical value to define the concordant and discordant Boolean matrices, which is more objective and accurate. Hence, the presented expected-HDD-based ELECTRE II method is more sophisticated and reasonable compared to the classical ELECTRE II method.

\subsubsection{Comparison with the Ideal-Based ELECTRE II Method}

The ideal-based ELECTRE II method was initially introduced by Liao et al. [42], in which the division of concordant and discordant sets is based on the positive and negative ideal solutions. In the following, we are going to apply this method to rank the given IT service outsourcers. For the sake of conciseness, only the pivotal and particular step results are shown below.

Let $\bar{h}_{S_{\phi}}$ and $\bar{h}_{S_{\phi}}{ }^{-}$be the DHHFL-PIS and DHHFL-NIS of the final decision matrix $\bar{R}$, respectively. According to the definition of the ideal solution introduced in Ref. [33], we can obtain

$$
\begin{aligned}
& \bar{h}_{S_{\phi}{ }^{*}}=\left(\left\{\zeta_{-1<\delta_{-0.3}>}, \zeta_{3<\delta_{0}>}\right\},\left\{\zeta_{-1<\delta_{-1.2}>}, \zeta_{-1<\delta_{-0.3}>}, \zeta_{3<\delta_{0}>}\right\},\right. \\
& \left.\left\{\zeta_{0<\delta_{-2.1}>}, \zeta_{0<\delta_{-0.8}>}, \zeta_{3<\delta_{0}>}\right\},\left\{\zeta_{0<\delta_{-2.9}>}, \zeta_{0<\delta_{-1.8}>}\right\},\left\{\zeta_{-1<\delta_{-0.5}>}, \zeta_{-1<\delta_{-0.5}>}, \zeta_{0<\delta_{-2}>}\right\}\right) \\
& \bar{h}_{S_{\phi}}{ }^{-}=\left(\left\{\zeta_{-2<\delta_{-0.8}>}, \zeta_{-2<\delta_{-0.5}>}\right\rangle,\left\{\zeta_{-2<\delta_{-1}>}, \zeta_{-2<\delta_{-0.8}>}, \zeta_{-2<\delta_{-0.3}>}\right\},\right. \\
& \left.\left\{\zeta_{-2<\delta_{-1.2}>}, \zeta_{-2<\delta_{-0.8}>}, \zeta_{-2<\delta_{-0.5}>}\right\rangle,\left\{\zeta_{-2<\delta_{-0.9}>}, \zeta_{-2<\delta_{-0.5}>}\right\rangle,\left\{\zeta_{-2<\delta_{-1}>}, \zeta_{-2<\delta_{-0.7}>}, \zeta_{-2<\delta_{-0.3}>}\right\}\right)
\end{aligned}
$$

Next, we calculate the distance between the DHHFLEs in with those in $\bar{h}_{S_{\phi}}{ }^{*}$ and $\bar{h}_{S_{\phi}}{ }^{-}$; the results are shown in Tables 11 and 12, respectively. 
Table 11. The distance between the DHHFL elements (DHHFLEs) in $\overline{\mathrm{R}}$ and $\bar{h}_{S_{\phi}}$.

\begin{tabular}{cccccc}
\hline$\left.-\bar{h}_{S_{\phi s j}} \boldsymbol{h}_{S_{\boldsymbol{\phi}}}{ }^{*}\right)$ & $j=1$ & $j=2$ & $j=3$ & $j=4$ & $j=5$ \\
\hline$s=1$ & 0.4253 & 0.2954 & 0.3054 & 0.0695 & 0.0319 \\
$s=2$ & 0.3461 & 0.3744 & 0.3594 & 0.1319 & 0.1207 \\
$s=3$ & 0 & 0.4018 & 0.4933 & 0.2450 & 0.2001 \\
$s=4$ & 0.5289 & 0 & 0 & 0 & 0.0271 \\
$s=5$ & 0.4345 & 0.2913 & 0.2900 & 0.0788 & 0.0205 \\
\hline
\end{tabular}

Table 12. The distance between the DHHFLEs in $\overline{\mathrm{R}}$ and $\bar{h}_{\mathcal{S}_{\phi}}{ }^{-}$.

\begin{tabular}{cccccc}
\hline $\boldsymbol{d}\left(\boldsymbol{h}_{S_{\phi s j}} \boldsymbol{h}_{\boldsymbol{S}_{\phi}}{ }^{-}\right)$ & $j=1$ & $j=2$ & $j=3$ & $j=4$ & $j=5$ \\
\hline$s=1$ & 0.1036 & 0.1064 & 0.1879 & 0.1756 & 0.1682 \\
$s=2$ & 0.1828 & 0.0274 & 0.1339 & 0.1132 & 0.0794 \\
$s=3$ & 0.5289 & 0 & 0 & 0 & 0 \\
$s=4$ & 0 & 0.4018 & 0.4933 & 0.2450 & 0.1731 \\
$s=5$ & 0.0944 & 0.1104 & 0.2034 & 0.1662 & 0.1796 \\
\hline
\end{tabular}

Then, the relative distance $\bar{d}$, defined in [33], can be obtained by the following equation:

$$
\bar{d}=\frac{d\left(\bar{h}_{S_{\phi s j}}, \bar{h}_{S_{\phi}{ }^{-}}\right)}{\left(d\left(\bar{h}_{S_{\phi s j}} \bar{h}_{S_{\phi}}\right)+d\left(\bar{h}_{S_{\phi s j}}, \bar{h}_{S_{\phi}}{ }^{-}\right)\right)} .
$$

The corresponding calculated results are presented in Table 13.

Table 13. The relative distance between the DHHFLEs in $\bar{R}$ and the ideal solution.

\begin{tabular}{cccccc}
\hline $\bar{d}$ & $j=1$ & $j=2$ & $j=3$ & $j=4$ & $j=5$ \\
\hline$s=1$ & 0.1959 & 1.1253 & 0.7849 & 1.1257 & 0.7953 \\
$s=2$ & 0.6282 & 1.7919 & 1.1182 & 1.1257 & 1.1286 \\
$s=3$ & 1.9932 & 1.9932 & 2.1182 & 2.1257 & 2.1286 \\
$s=4$ & 2.1282 & 1.6938 & 1.0256 & 0.1257 & 1.1286 \\
$s=5$ & 1.1282 & 1.1253 & 0.7849 & 1.1257 & 0.7953 \\
\hline
\end{tabular}

On the basis of the partition rules of the concordant and discordant sets, we can obtain the corresponding strong, moderate, and weak concordant sets, the strong, moderate, and weak discordant sets, and the indifferent sets, respectively.

Similarly to our proposed approach, the next step is to reckon on the DHHFLCI and DHHFLDI and to compare them to the critical values $\bar{C}$ and $\bar{D}$. Then, we can get the concordant and discordant Boolean matrices as

$$
B_{C}=\left[b c_{s t}\right]_{5 \times 5}=\left[\begin{array}{ccccc}
- & 1 & 1 & 0 & 0 \\
0 & - & 1 & 0 & 0 \\
1 & 0 & - & 1 & 1 \\
1 & 1 & 1 & - & 1 \\
1 & 1 & 1 & 0 & -
\end{array}\right], B_{D}=\left[b d_{s t}\right]_{5 \times 5}=\left[\begin{array}{ccccc}
- & 0 & 0 & 0 & 0 \\
0 & - & 0 & 0 & 0 \\
1 & 1 & - & 0 & 1 \\
1 & 1 & 0 & - & 1 \\
1 & 0 & 0 & 0 & -
\end{array}\right] .
$$

Then, the overall matrix can be given as $B=\left[b c_{s t} \times b d_{s t}\right]_{5 \times 5}=\left[\begin{array}{ccccc}- & 0 & 0 & 0 & 0 \\ 0 & - & 0 & 0 & 0 \\ 1 & 0 & - & 0 & 1 \\ 1 & 1 & 0 & - & 1 \\ 1 & 0 & 0 & 0 & -\end{array}\right]$. 
Finally, the outranking graph is drawn as in Figure 9.

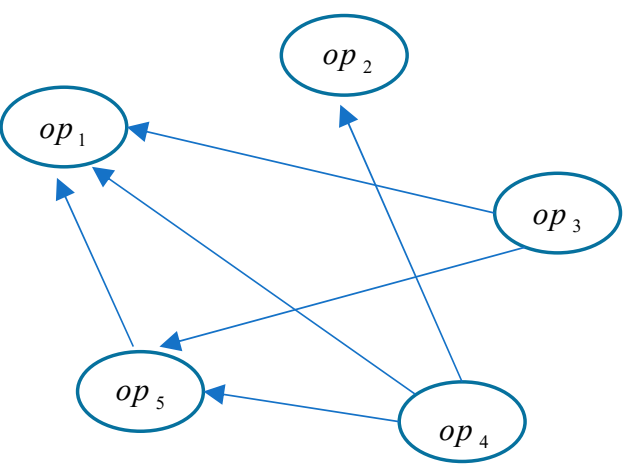

Figure 9. The outranking graph.

As seen in Table 14, the ranking results obtained by the ideal-based ELECTRE II method are distinct from those derived by the expected-HDD-based ELECTRE II approach. In particular, the relation between $o p_{2}, o p_{3}$ and $o p_{3}, o p_{4}$ is indeterminate in Figure 9, which can be explicitly determined by our proposed method. Next, two main reasons are given for this inconsistency:

Table 14. The ranking results.

\begin{tabular}{cc}
\hline Method & Ranking \\
\hline Proposed method & $o p_{4}>o p_{5}>o p_{1}>o p_{2}>o p_{3}$ \\
$o p_{4}>o p_{5}>o p_{1} ;$
\end{tabular}

(1) The ideas or the core points of these two methods are somewhat distinct. The ideal-based ELECTRE II method introduced by Liao et al., as the name implies, is an improved ELECTRE II method based on the ideal solution. The method believes that the smaller the difference with PIS and, simultaneously, the greater the difference with NIS, the more positive the evaluation value is, which is reasonable to some extent. However, without taking the variance into account, the determination of the ideal solution is apt to be influenced by the extreme value, which can be illustrated by the following example.

Example 3. Suppose that $h_{S_{\phi s j}}=\left\{\zeta_{-2<\delta_{-3}>}, \zeta_{1<\delta_{1}>}\right\}$ and $h_{S_{\phi t j}}=\left\{\zeta_{-1<\delta_{-1}>}, \zeta_{1<\delta_{1}>}\right\}$ are the evaluation values of the options op $p_{s}$ and $o_{t}$ under the criterion $\mathrm{cr}_{j}$. According to the definition of the ideal solution introduced

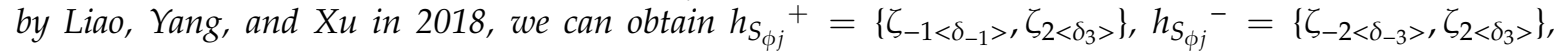
$d\left(h_{S_{\phi s j}}, h_{S_{\phi j}}{ }^{+}\right)=d\left(h_{S_{\phi t j}}, h_{S_{\phi j}}{ }^{+}\right)=0.139$ and $d\left(h_{S_{\phi s j}} h_{S_{\phi j}}{ }^{-}\right)=d\left(h_{S_{\phi t j}}, h_{S_{\phi j}}{ }^{-}\right)=0.139$. On the basis of the idea of the ideal-based ELECTRE II method, it can be concluded that op $p_{s}$ is indifferent with opt under the attribute $c r$. Nonetheless, in line with the comparison rule of DHHFLEs, op $p_{s}$ is actually superior to opt owing to its smaller (hesitant) deviation degree.

Obviously, the ranking result obtained by the ideal-based method may be inaccurate under situations similar to the abovementioned. Instead, our proposed method is based on the improved comparison rule of DHHFLEs, which is well considered and proven to be accurate.

(2) Compared to the ELECTRE II method introduced by Liao et al., our proposed method can address such issues where there are pairs of alternatives that are incomparable or the differences between them are not conspicuous. This is because our proposed method can locate the unreasonable position in the Boolean matrix and modify it; then, the weak outranking relation between the corresponding alternatives can be established. However, the ideal-based ELECTRE II method ignores the weak priority relation between two seemingly incomparable solutions. From a problem-solving perspective, 
our primary task is to determine the priority relationship between the two options, not the degree of priority between them. Hence, our presented method is more practical and effective than the ideal-based ELECTRE II method.

In a word, by virtue of the expected values and the HDDs of the evaluations, our presented method can take both the hesitant degree and the deviation degree into consideration and can eliminate the effect of the unreasonable value. In addition, it can identify the weak outranking relation between actions that are seemingly incomparable or where the differences between them are not conspicuous. Therefore, the proposed method is more reasonable and applicable to practice than some existing ELECTRE II methods.

\section{Conclusions}

With the deepening of the fourth industrial revolution, operations/production, supply-chain, management, and governance, as well as products and services, will be increasingly transformed and reshaped [47-49]. In this process, outsourcing public services has become an effective means for the government to improve service quality and reduce operating costs. In the process of outsourcing, choosing the most appropriate service provider is a pivotal issue for governments. By extending the ELECTRE II method to the DHHFL environment, we proposed a novel expected-HDD-based ELECTRE II method to tackle the PSO selection problem. The core content of this paper is shown as follows:

1. A new comparison method for DHHFLEs is proposed based on the definition of the expected value and the HDD of a DHHFLE, through which the magnitude relationship between any two alternatives under a specific criterion can be clearly determined.

2. Motivated by the PA operator, we present the support degree of a DHHFLE and put forward a novel weight-determination method for experts with respect to each criterion. In addition, based on the multi-objective optimization, a weight-determination method for criteria is introduced to handle the case where the criterion's weights are completely unknown.

3. Combining the new comparison method and the weight-determination methods for experts and criteria, an improved DHHFL-ELECTRE II is constructed to deal with the problem of PSO selection. In addition, from the perspective of information form and decision-making processes, our method has universality in dealing with MCDM problems, and provides a simple, efficient, and convenient way to address the individual preferences of DMs.

4. Comparisons between the proposed method and existing methods, such as the method based on the PA operator, the classical ELECTRE II method, and the ideal-based ELECTRE II method, are given to illustrate that our method can identify more reasonable outcomes than the existing methods.

For the direction of our future research, our ideas are as follows: Firstly, for the DHHFLTS, the semantic difference between two adjacent linguistic terms is equal, which cannot reflect the preferences of DMs. Hence, we are going to conduct an in-depth study of unbalanced DHLTS [50]. Secondly, taking the psychology of people into account, we will consider a combination of regret theory [51] for multi-attribute decision making and using the regret-rejoicing function, which aims at reducing the regret value of experts, to measure the performance of alternatives.

Author Contributions: Conceptualization, Z.L. and P.L.; Formal analysis, D.W.; Funding acquisition, P.L.; Methodology, X.Z., X.W. and D.W.; Software, L.L.; Supervision, L.L. and X.W.; Writing-original draft, X.Z. All authors have read and agreed to the published version of the manuscript.

Funding: This research was funded by the Humanities and Social Sciences Research Project of Ministry of Education of China (No. 17YJA630065), the Shandong Provincial Natural Science Foundation of China (No. ZR2017MG007), the Project of Shandong Province Higher Educational Science and Technology Program (No. J16LN25), the Special Funds of Taishan Scholars Project of Shandong Province (No. ts201511045), and the National Science Foundation of China (No.71771140).

Conflicts of Interest: The authors declare no conflict of interest. 


\section{References}

1. Yager, R.R. The power average operator. IEEE Trans Syst. Man Cybern. Part A Syst. Hum. 2001, 31, 724-731. [CrossRef]

2. Yun, J.J.; Park, K.B.; Hahm, S.D. Basic income with high open innovation dynamics: The way to the entrepreneurial state. J. Open Innov. Technol. Mark. Complex. 2019, 5, 41. [CrossRef]

3. Yun, J.H.J.; Won, D.K.; Park, K. Dynamics from open innovation to evolutionary change. J. Open Innov. Technol. Mark. Complex. 2016, 2, 7. [CrossRef]

4. Yun, J.H.J.; Won, D.K.; Park, K.B. Entrepreneurial cyclical dynamics of open innovation. J. Evol. Econ. 2018, 28, 1151-1174. [CrossRef]

5. Kim, Y.; Lee, M. Typology and Unified Model of the Sharing Economy in Open Innovation Dynamics. J. Open Innov. Technol. Mark. Complex. 2019, 5, 102. [CrossRef]

6. Oganisjana, K.; Kozlovskis, K. The Identification of Opportunities for Innovations through Collecting Problems from Citizens. J. Open Innov. Technol. Mark. Complex. 2019, 5, 76. [CrossRef]

7. Lee, I.; Kim, E. Factors Affecting the Outbound Open Innovation Strategies in Pharmaceutical Industry: Focus on Out-Licensing Deal. J. Open Innov. Technol. Mark. Complex. 2019, 5, 73. [CrossRef]

8. Yun, J.H.J. How do we conquer the growth limits of capitalism? Schumpeterian Dynamics of Open Innovation. J. Open Innov. Technol. Mark. Complex. 2015, 1, 17. [CrossRef]

9. Golooba-Mutebi, F. Devolution and outsourcing of municipal services in Kampala city, Uganda: An early assessment. Public Adm. Dev. Int. J. Manag. Res. Pract. 2003, 23, 405-418. [CrossRef]

10. Moon, J.; Choe, Y.C.; Chung, M.; Jung, G.H.; Swar, B. IT outsourcing success in the public sector: Lessons from e-government practices in Korea. Infdev 2016, 32, 142-160. [CrossRef]

11. Samsudin, N.M.; Hashim, R.; Fuzi, S.F.S.M. Electronic government outsourcing issues in Malaysia. J. Outsourc. Organ. Inf. Manag. 2013, 2013, 1. [CrossRef]

12. Aman, A.C., Jr. An administrative law perspective on government social service contracts: Outsourcing prison health care in New York City. Indiana J. Glob. Leg. Stud. 2007, 14, 301-328. [CrossRef]

13. Heinecken, L. Outsourcing public security: The unforeseen consequences for the military profession. Armed Forces Soc. 2014, 40, 625-646. [CrossRef]

14. Peng, H.G.; Shen, K.W.; He, S.S.; Zhang, H.Y.; Wang, J.Q. Investment risk evaluation for new energy resources: An integrated decision support model based on regret theory and ELECTRE III. Energy Convers. Manag. 2019, 183, 332-348. [CrossRef]

15. Zadeh, L.A. Fuzzy sets. Infect. Control. 1965, 8, 338-353. [CrossRef]

16. Mizumoto, M.; Tanaka, K. Some properties of fuzzy sets of type 2. Infect. Control. 1976, 31, 312-340. [CrossRef]

17. Atanassov, K.T. More on intuitionistic fuzzy sets. Fuzzy Sets Syst. 1989, 33, 37-45. [CrossRef]

18. Torra, V. Hesitant fuzzy sets. Int. J. Intell. Syst. 2010, 25, 529-539. [CrossRef]

19. Rodriguez, R.M.; Martinez, L.; Herrera, F. Hesitant fuzzy linguistic term sets for decision making. IEEE Trans. Fuzzy Syst. 2011, 20, 109-119. [CrossRef]

20. Martı, L.; Herrera, F. An overview on the 2-tuple linguistic model for computing with words in decision making: Extensions, applications and challenges. Inf. Sci. 2012, 207, 1-18.

21. Pang, Q.; Wang, H.; Xu, Z. Probabilistic linguistic term sets in multi-attribute group decision making. Inf. Sci. 2016, 369, 128-143. [CrossRef]

22. Gou, X.; Liao, H.; Xu, Z.; Herrera, F. Double hierarchy hesitant fuzzy linguistic term set and MULTIMOORA method: A case of study to evaluate the implementation status of haze controlling measures. Inf. Fusion 2017, 38, 22-34. [CrossRef]

23. Gou, X.; Xu, Z.; Liao, H.; Herrera, F. Multiple criteria decision making based on distance and similarity measures under double hierarchy hesitant fuzzy linguistic environment. Comput. Ind. Eng. 2018, 126, 516-530. [CrossRef]

24. Liu, X.; Wang, X.; Qu, Q.; Zhang, L. Double hierarchy hesitant fuzzy linguistic mathematical programming method for MAGDM based on Shapley values and incomplete preference information. IEEE Access 2018, 6, 74162-74179. [CrossRef]

25. Gou, X.; Xu, Z.; Herrera, F. Consensus reaching process for large-scale group decision making with double hierarchy hesitant fuzzy linguistic preference relations. Knowl. Based Syst. 2018, 157, 20-33. [CrossRef] 
26. Krishankumar, R.; Subrajaa, L.S.; Ravichandran, K.S.; Kar, S.; Saeid, A.B. A Framework for Multi-Attribute Group Decision-Making Using Double Hierarchy Hesitant Fuzzy Linguistic Term Set. Int. J. Fuzzy Syst. 2019, 21, 1130-1143. [CrossRef]

27. Wei, C.C.; Cheng, Y.L.; Lee, K.L. How to select suitable manufacturing information system outsourcing projects by using TOPSIS method. Int. J. Prod. Res. 2019, 57, 4333-4350. [CrossRef]

28. Yadav, G.; Mangla, S.K.; Luthra, S.; Jakhar, S. Hybrid BWM-ELECTRE-based decision framework for effective offshore outsourcing adoption: A case study. Int. J. Prod. Res. 2018, 56, 6259-6278. [CrossRef]

29. Liu, Z.; Liu, P.; Liang, X. Multiple attribute decision-making method for dealing with heterogeneous relationship among attributes and unknown attribute weight information under q-rung orthopair fuzzy environment. Int. J. Intell. Syst. 2018, 09, 1900-1928. [CrossRef]

30. Ji, P.; Zhang, H.Y.; Wang, J.Q. Selecting an outsourcing provider based on the combined MABAC-ELECTRE method using single-valued neutrosophic linguistic sets. Comput. Ind. Eng. 2018, 120, 429-441. [CrossRef]

31. Benayoun, R.; Roy, B.; Sussman, B. ELECTRE: Une méthode pour guider le choix en présence de points de vue multiples. In Note de Travail 49, SEMA-METRA International, Direction Scientifique; KAP: Berlin, Germany, 1966.

32. Hashemi, H.; Mousavi, S.M.; Zavadskas, E.K.; Chalekaee, A.; Turskis, Z. A New Group Decision Model Based on Grey-Intuitionistic Fuzzy-ELECTRE and VIKOR for Contractor Assessment Problem. Sustainability 2018, 10, 1635. [CrossRef]

33. Micale, R.; Giallanza, A.; Russo, G.; La Scalia, G. Selection of a Sustainable Functional Pasta Enriched with Opuntia Using ELECTRE III Methodology. Sustainability 2017, 9, 885. [CrossRef]

34. Hokkanen, J.; Salminen, P.; Rossi, E.; Ettala, M. The choice of a solid waste management system using the ELECTRE II decision-aid method. Waste Manag. Res. 1995, 13, 175-193. [CrossRef]

35. Devadoss, A.V.; Rekha, M. A New Intuitionistic Fuzzy ELECTRE II approach to study the Inequality of women in the society. Glob. J. Pure Appl. Math. 2017, 13, 6583-6594.

36. Wen, Z.; Yu, Y.; Yan, J. Best available techniques assessment for coal gasification to promote cleaner production based on the ELECTRE-II method. J. Clean. Prod. 2016, 129, 12-22. [CrossRef]

37. Chen, N.; Xu, Z. Hesitant fuzzy ELECTRE II approach: A new way to handle multi-criteria decision making problems. Inf. Sci. 2015, 292, 175-197. [CrossRef]

38. Pan, L.; Ren, P.; Xu, Z. Therapeutic schedule evaluation for brain-metastasized non-small cell lung cancer with a probabilistic linguistic ELECTRE II method. Int. J. Environ. Res. 2018, 15, 1799. [CrossRef]

39. Vinodh, S.; Girubha, R.J. Sustainable concept selection using ELECTRE. Clean Technol. Environ. Policy 2012, 14, 651-656. [CrossRef]

40. Liu, Z.; Wang, S.; Liu, P. Multiple attribute group decision making based on q-rung orthopair fuzzy Heronian mean operators. Int. J. Intell. Syst. 2018, 12, 2341-2363. [CrossRef]

41. Wan, S.P.; Xu, G.L.; Dong, J.Y. Supplier selection using ANP and ELECTRE II in interval 2-tuple linguistic environment. Inf. Sci. 2017, 385, 19-38. [CrossRef]

42. Liao, H.C.; Yang, L.Y.; Xu, Z.S. Two new approaches based on ELECTRE II to solve the multiple criteria decision making problems with hesitant fuzzy linguistic term sets. Appl. Soft Comput. 2018, 63, 223-234. [CrossRef]

43. Lin, M.; Chen, Z.; Liao, H.; Xu, Z. ELECTRE II method to deal with probabilistic linguistic term sets and its application to edge computing. Nonlinear Dyn. 2019, 96, 2125-2143. [CrossRef]

44. Figueira, J.R.; Greco, S.; Roy, B.; Słowiński, R. ELECTRE Methods: Main Features and Recent Developments. In Handbook of Multicriteria Analysis; Springer: Berlin/Heidelberg, Germany, 2010; pp. 51-89.

45. Ju, Y. A new method for multiple criteria group decision making with incomplete weight information under linguistic environment. Appl. Math. Model. 2014, 38, 5256-5268. [CrossRef]

46. Wei, G.; Lu, M. Pythagorean fuzzy power aggregation operators in multiple attribute decision making. Int. J. Intell. Syst. 2018, 33, 169-186. [CrossRef]

47. Pyka, A.; Bogner, K.; Urmetzer, S. Productivity Slowdown, Exhausted Opportunities and the Power of Human Ingenuity—Schumpeter Meets Georgescu-Roegen. J. Open Innov. Technol. Mark. Complex. 2019, 5, 39. [CrossRef]

48. Cooke, P. World turned upside down: Entrepreneurial decline, its reluctant myths and troubling realities. J. Open Innov. Technol. Mark. Complex. 2019, 5, 22. [CrossRef] 
49. Lee, M.H.; Yun, J.H.J.; Pyka, A. How to respond to the fourth industrial revolution, or the second information technology revolution? Dynamic new combinations between technology, market, and society through open innovation. J. Open Innov. Technol. Mark. Complex. 2018, 4, 21. [CrossRef]

50. Fu, Z.; Liao, H. Unbalanced double hierarchy linguistic term set: The TOPSIS method for multi-expert qualitative decision making involving green mine selection. Inf. Fusion 2019, 51, 271-286. [CrossRef]

51. Connolly, T.; Zeelenberg, M. Regret in decision making. Curr. Dir. Psychol. Sci. 2002, 11, 212-216. [CrossRef]

(C) 2020 by the authors. Licensee MDPI, Basel, Switzerland. This article is an open access article distributed under the terms and conditions of the Creative Commons Attribution (CC BY) license (http://creativecommons.org/licenses/by/4.0/). 